\title{
Space Project for Astrophysical and Cosmological Exploration (SPACE), an ESA stand-alone mission and a possible contribution to the Origins Space Telescope
}

\author{
Denis Burgarella, et al. [full author details at the end of the article]
}

Received: 7 August 2020 / Accepted: 28 January 2021 / Published online: 18 July 2021

(c) The Author(s) 2021

\begin{abstract}
We propose a new mission called Space Project for Astrophysical and Cosmological Exploration (SPACE) as part of the ESA long term planning Voyage 2050 programme. $S P A C E$ will study galaxy evolution at the earliest times, with the key goals of charting the formation of the heavy elements, measuring the evolution of the galaxy luminosity function, tracing the build-up of stellar mass in galaxies over cosmic time, and finding the first super-massive black holes (SMBHs) to form. The mission will exploit a unique region of the parameter space, between the narrow ultra-deep surveys with HST and JWST, and shallow wide-field surveys such as the Roman Space Telescope and EUCLID, and should yield by far the largest sample of any current or planned mission of very high redshift galaxies at $z>10$ which are sufficiently bright for detailed follow-up spectroscopy. Crucially, we propose a widefield spectroscopic near-IR + mid-IR capability which will greatly enhance our understanding of the first galaxies by detecting and identifying a statistical sample of the first galaxies and the first supermassive black holes, and to chart the metal enrichment history of galaxies in the early Universe - potentially finding signatures of the very first stars to form from metal-free primordial gas. The wide-field and wavelength range of SPACE will also provide us a unique opportunity to study star formation by performing a wide survey of the Milky Way in the near-IR + mid-IR. This science project can be enabled either by a stand-alone ESA-led M mission or by an instrument for an L mission (with ESA and/or NASA, JAXA and other international space agencies) with a wide-field (sub-)millimetre capability at $\lambda>500$ $\mu \mathrm{m}$.
\end{abstract}

Keywords Extragalactic astrophysics · Cosmology · First stars · First dust grains · First galaxies $\cdot$ Reionization

\footnotetext{
Additional authors with respect to the SPACE Projects: some of the people now listed in the paper have been involved in the preparation of this project either scientifically or instrumentally. But they did not gave the first author the authorization to add their name to the document submitted to ESA soon enough. However, after submitting the project to ESA, they send their agreement and they are now included in the author list.
} 


\section{Introduction}

There has been enormous progress over the past decade in discovering galaxies which existed early in the history of the Universe (within a billion years of the Big Bang, at $z>6$ ), thanks in large part to images from the Hubble Space Telescope, and confirming spectroscopy from large telescopes on the ground. The next few years will see the "high redshift frontier" pushed even further with the James Webb Space Telescope (JWST, [37]) and ground-based Extremely Large Telescopes (ELTs).

However, the limited field of view of these facilities (especially JWST), and sensitivity only out to the near-infrared (near-IR, $\lambda<2 \mu \mathrm{m}$ ) for the Roman Space Telescope (formerly WFIRST, [23]) and EUCLID [49] wide-field imaging space missions, mean that a crucial piece of the jigsaw remains missing: a wide-field imaging survey, working at near and mid-IR wavelengths (necessarily from space) is needed to find the very rare most massive and luminous galaxies at the highest redshifts, the progenitors of which are likely to be the first galactic structures to form. NIR spectroscopy at $\lambda>2 \mu \mathrm{m}$ (corresponding to the rest-frame optical frame) is also mandatory to get complete information (metallicity, stellar mass) for galaxies at $\mathrm{z}>10$.

The landscape of astrophysics in the timeframe from 2035-2050 is expected to be very rich: the JWST mission will have been completed, presumably finding a wealth of faint galaxies at high redshift and addressing the role of these early galaxies in the reionization of the inter-galactic medium. ALMA [94] will be a very mature facility by then and SKA [39] will have explored the molecular emission and dust re-emission from some of these objects. The re-ionisation of the Universe was achieved by low luminosity sources [11, 14, 15, 65, 69]. These low luminosity sources would only be visible if they are in groups or proto clusters [21]. This is likely so for the first galaxies, which were of very low luminosity. Thus, detecting proto clusters from $\mathrm{z} \sim 6$ to $\mathrm{z} \sim 15$ would unveil the history of the Universe's reionisation [19, 67]. Rare and bright sources at high redshift (as well as transients such as distant supernovae) will be explored by the Rubin Observatory (previously LSST, [43]) on the ground, and EUCLID and the Roman Space Telescope in space, at wavelengths below 2 microns. In the X-ray, after a hiatus of many decades new facilities such as Athena [6] will see AGN out to unprecedented distances. But there is a key gap in the parameter space that remains unexploited - a wide-field IR survey mission with spectroscopy and imaging working beyond 2 microns that we propose to address here.

Our proposed SPACE mission will place the ESA community in a leading position to study the early Universe after JWST's deep pencil beam surveys via an unbiased census of the first-light objects. The SPACE mission will also provide us with the high angular resolution survey of the Milky Way in the near-IR and mid-IR to statistically study the star formation phenomena in the Milky Way. SPACE's widefield imaging and integral-field / multi-object spectroscopy in space are the major instrumental breakthroughs that will enable this new window in astronomy. 


\section{Extragalactic astrophysics and cosmology}

Our main objectives for extragalactic astrophysics are related to the birth of the first galaxies and AGN, and to the rise of the metals in the Universe.

\subsection{Key questions}

\subsubsection{When and how did galaxies form?}

The SPACE mission will achieve photometric identification of primordial star forming galaxies at $10<\mathrm{z}<15$, i.e. less than 0.3 to 0.5 Gyr after the Big Bang, over much larger volumes than available to HST or JWST. The galaxies will be selected through the Lyman break technique (e.g. [11, 14, 16, 81]) where intervening neutral hydrogen absorbs all light at wavelengths short-ward of Ly $\alpha 1216 \AA$ in the rest-frame, causing the highest-redshift objects to "drop out" of visible and near-IR images. The expected density of these galaxies at $\mathrm{z}>14$ is estimated to be $\sim 1 \mathrm{deg}^{-2}$ at $\mathrm{m}_{\mathrm{AB}}=$ 28 (e.g. [18]). To detect a statistically significant sample at the highest redshifts, an imaging survey of about $200 \mathrm{deg}^{2}$ is necessary. Several hundred sources are needed so as to determine the luminosity function and its evolution with redshift (particularly the shape at the bright end to probe the effects of feedback at early times). This sample of luminous galaxies will be the largest one obtained with any facility because: (1) JWST is unlikely to build surveys over areas much larger than $\sim 1 \mathrm{deg}^{2}$, i.e., HST-like which means that the probability of detecting such luminous galaxies is low at $\mathrm{z}>12$ and, (2) detecting these primordial galaxies requires any facility to have at least 2 bands at $\lambda>1.3 \mu \mathrm{m}($ at $\mathrm{z}=10)$ and $\lambda>2.0 \mu \mathrm{m}($ at $\mathrm{z}=15)$, to provide robust colours at wavelengths beyond the Lyman break for photometric redshifts. To date, only JWST and SPACE have the appropriate wavelength range extending beyond $2.0 \mu \mathrm{m}$ with the required sensitivity.

\subsubsection{Spectroscopic detection and identification of photometrically faint emission line galaxies via a blind spectroscopic survey (with integral-field spectroscopy)}

Imaging surveys allow the detection of galaxies with a strong continuum. However, an important subset of galaxies, younger (and therefore at low mass compared to today's standard) and undergoing strong star-bursting events, are optimally detected via spectroscopic surveys aiming at strong emission lines. VLT MUSE's results [3] discovered many Ly $\alpha$ emitting galaxies that are completely undetected in the HST deep images down to $\mathrm{I}(\mathrm{F} 814 \mathrm{~W})>29.5$. These sources that have no HST counterparts represent $30 \%$ of the entire Ly $\alpha$ emitter sample. A blind and relatively widefield integral-field spectroscopic (IFS) survey is the unique way to detect these objects - and provides a great sensitivity advantage over "slitless" spectroscopy, even from space, where each pixel records background noise at all wavelengths. No other current or planned space facility will feature an IFS instrument with such a large field-of-view. SPACE's IFS will build a survey via parallel observations and 
reach magnitudes as deep as the widest JWST NIRSpec survey (e.g. in the JWST Wide Field survey as defined in [56], in the F115W and F220W filters) but over a much larger area of more than $1 \mathrm{deg}^{2}$ (ten times the area over which the anticipated NIRSpec surveys will draw their targets from). Moreover, JWST/NIRSpec will not obtain spectroscopy over its entire field of view (except in the NIRCAM and NIRISS slitless grism mode but still on small fields of about 2.2' x 2.2'): a prior photometric detection is needed to define the slits for the micro-shutter arrays (and the alternative IFS mode on NIRSpec covers $3 \times 3 \operatorname{arcsec}^{2}$, only $0.03 \%$ of the full field of the NIRSpec micro-shutter array). IFS with SPACE will look for signatures of the first generation of Population III stars in the earliest galaxies, chart the evolution of metal enrichment and the assembly of mass in galaxies over cosmic time.

\subsubsection{The first quasars and massive black holes}

The density of very high redshift $(z>6)$ luminous quasars is very low, with only a handful currently known at $z>7$, although the very existence of supermassive black holes at these early times presents strong challenges to seed formation and blackhole growth models. Crucially, SPACE has the wavelength coverage to identify quasars out to high redshift, and SPACE's field-of-view is large enough to directly detect $\sim 100$ quasars at $\mathrm{z}>8$ pushing down to fainter luminosities than current surveys. Moreover, we will provide sufficient time in SPACE's observation schedule to observe photometrically and spectroscopically these quasars into the rest-frame optical. This will provide unique information on the early co-evolution of galaxies and supermassive black holes but also, this will allow the intergalactic medium to be studied along the line of sight. The IFS will be very valuable to try and detect the environment of these early supermassive black holes.

\subsubsection{The birth of metals}

Big Bang nucleosynthesis creates hydrogen and helium, but all elements heavier than beryllium are formed later in stars and supernovae. Hence the "metallicity" (the enrichment of cosmic gas by heavy elements) acts as a clock - the elemental abundances are built up with time with successive generations of star formation. Emission line diagnostics have been used at intermediate redshifts to determine the metallicity of the star- forming gas, and a key probe of galaxy evolution is the massmetallicity relation (e.g. [85]), which indicates how star formation and chemical enrichment proceed in galaxies as a function of galaxy stellar mass. Good determinations have been made at low redshift (e.g. SDSS, [54, 88]) and more recent work at intermediate redshifts (e.g. [96] at z 1). Metallicity measurements are currently limited to $\mathrm{z} \lesssim 3$ due to spectral coverage from the ground, and there exists a small number of $\mathrm{z} \sim 3$ estimates (e.g. [52, 53]). The slope and offset of the mass-metallicity relation will evolve with redshift if the characteristic timescale (or efficiency) of chemical enrichment depends on the stellar mass. At the higher redshifts there appears to be significant differences between the current small number of observations and theoretical predictions from chemical evolution models (e.g. [25, 84]). 


\subsection{Detailed science case}

\subsubsection{The first galaxies}

Our understanding of how galaxies form and evolve over the last 13 Gyrs of cosmic time has increased dramatically over the last decades (e.g. see reviews [30, 51, 68]). Tremendous effort has been invested in finding and studying the most distant sources, particularly since they offer stringent constraints on galaxy formation, allowing us to test models of early galaxy formation and evolution (e.g. [46, 72, 91]), along with studying the epoch of reionization (e.g. [27, 42, 74]). Nevertheless, some fundamental questions remain regarding the very early Universe. Where and when did the first stars and black holes form? What were their properties? Can we finally identify and characterise them, thus confronting state-of-the-art predictions and pushing models much further? While ultra-deep surveys with HST have been extremely successful, most of the small number of current $z=10$ candidates have not been found in the Hubble Ultra Deep Field but in wider-field, shallower HST surveys. We now have growing evidence that the bright end of the luminosity function is evolving less rapidly than the faint end (e.g. [12]), which is not well captured or reproduced in current models. This motivates a search for bright galaxies out to high redshift to accurately determine this evolution. The unique combination of depth, area, and wavelength coverage provided by the imaging camera on SPACE will provide the first significant searches for $\mathrm{z}>10$ galaxies (potentially revealing hundreds of high redshift sources with star formation rates greater than $100 \mathrm{M}_{\odot} \mathrm{yr}^{-1}$ ), for the first quasars at $\mathrm{z}>7$, and for massive evolved galaxies at $\mathrm{z}>$ 6. With these searches, we can constrain unexpected physics and test the $\Lambda \mathrm{CDM}$ paradigm. Complementary to this imaging, the wide-field integral field spectroscopy (IFS) capability of SPACE offers an unprecedented opportunity to study very young and low-mass strongly star-bursting proto-galaxies in the early Universe, to search for the elusive first generation of stars (Population III), and to chart the chemical enrichment history and mass assembly of galaxies at early times.

The high redshift frontier The brightest most massive galaxies at high redshift occupy a unique region in parameter space which provides a new test of galaxy formation physics in a different regime to that probed by the small-area surveys of HST and JWST. At $\mathrm{z}=13-15$, the Universe will have an age of only 250 - 300 million years. These early times are as yet unexplored, and it is unlikely that JWST will survey sufficient area to identify even one such galaxy near the bright end of the luminosity function (LF), while surveys like Euclid or the Roman Space telescope are limited to $\lambda<2 \mu \mathrm{m}$ and do not have sufficiently red filters to identify such galaxies at all (Fig. 1). Pushing to these hitherto-unexplored highest redshifts is key to understanding when the first stars and galaxies formed.

Large samples of robust candidates at $\mathrm{z} \sim 11-12 S P A C E$ is also likely to provide the community with the largest and most complete sample of ultra-bright galaxies at $\mathrm{z}=11-12$. While sources at these redshifts can, in principle, be selected based on the Roman Space telescope survey (the Euclid survey will likely be too shallow at 


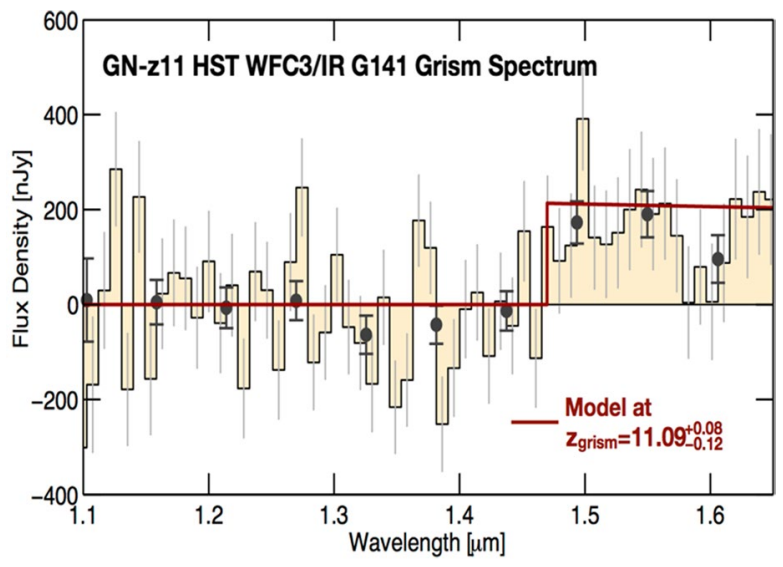

Fig. 1 The Spectral Energy Distribution of the galaxy discovered at $z=11.09$ [63] presents a break around $\lambda=1.5 \mu \mathrm{m}$ due to the intervening Lyman-alpha forest absorption. To get a reliable estimate of the redshifts in the $10<\mathrm{z}<15$ range, it is crucial to collect data at wavelengths above and below the break. The NIR+MIR range is therefore needed. This is JWST's choice and also that of SPACE. Other facilities with limited capabilities beyond $\sim 2 \mu \mathrm{m}$ will not be suitable for this quest - two or more filters longward of the break are needed for secure identification. Credit: P. Oesch / Observatoire de Genève

$\mathrm{z}>10$ ), rare $\mathrm{z} \sim 11-12$ galaxies will be difficult to distinguish from the much more ubiquitous contaminant population due to the lack of filters beyond $2 \mu \mathrm{m}$ (and hence restricted colour information), making any confirmation campaign extremely time expensive. The SPACE mission overcomes these significant issues with contamination and expensive follow-up confirmation.

Most of the high redshift objects lack spectroscopic redshifts, with the luminosity functions derived just from LBG candidates identified in images. Accurate spectroscopic redshifts are critical, since the interloper fraction of lower-redshift sources is very uncertain. Critically, the inferred luminosity function depends strongly on the calculation of the volume sampled, which in turn depends on the selection and completeness (Fig. 2). These are strongly affected by the redshift distribution and spectral slopes of the Lyman-break galaxies.

In order to effectively select Lyman break galaxies at high redshift, we will use optical imaging to reject low redshift red contaminants in our fields (which would be detected at shorter wavelength, unlike the highest redshift objects). The planned Rubin Observatory deep-drilling fields will cover $\sim 50-100 \mathrm{deg}^{2}$ and could be expanded further to match our intended survey area. The anticipated depths of $\mathrm{m}_{\mathrm{AB}}$ $=28$ at $5 \sigma$ (equivalent to a $2 \sigma$ upper limit of $\mathrm{m}_{\mathrm{AB}}=29$ ) are sufficient to cleanly select Lyman break galaxies with $\mathrm{m}_{\mathrm{AB}}=28$ at wavelengths beyond Lyman-alpha, particularly when the ugrizy images are co-added for greater depth. Alternatively, the existing HyperSuprimeCam on Subaru will reach comparable depths in $g$ and $r$ band in the planned Ultra Deep survey ( 2.4 nights per pointing). A survey in a single band with Subaru over SPACE's 200deg 2 area would take about 260 nights.

Making use of published predictions from Mason et al. [56], Behroozi and Silk [8] and Mashian et al. [55], we predict we would find $\sim 5000$ at $\mathrm{z} \sim 13$ galaxies, $\sim 200$ 


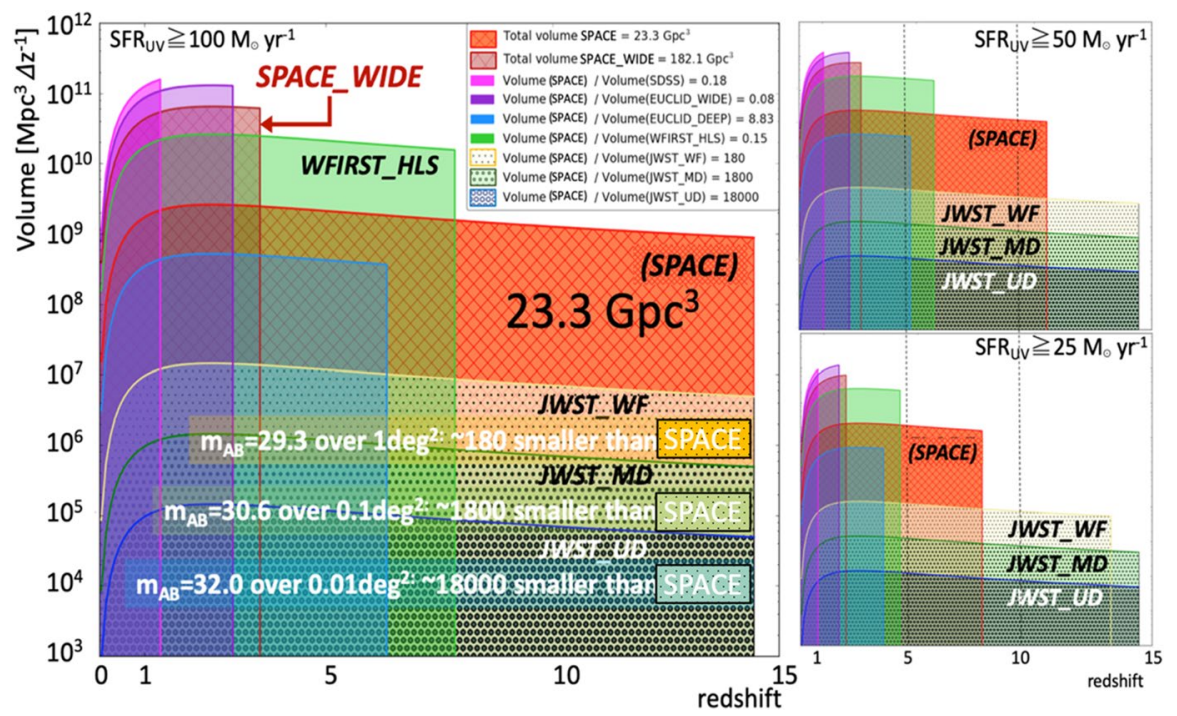

Fig 2 The volume of the Universe for different projects in which galaxies with various star formation rate thresholds (from the rest-frame UV) can be detected. The legend gives SPACE's volume and the ratio of the other projects with respect to SPACE. None of the JWST surveys will have comoving volumes comparable to lower redshift ones $(\mathrm{z}<6)$. On the other hand, SPACE's $200 \mathrm{deg}^{2}$ survey at $\mathrm{z}>8$ will reach about $10 \%$ the volume of the Euclid WIDE and Roman Space telescope HLS surveys at much lower redshift. Even at lower SFRs, i.e. normal star-forming galaxies, SPACE will be able to cover a huge volume unreachable for any other survey

galaxies at $\mathrm{z} \sim 14$ galaxies, and still a few tens of galaxies at $\mathrm{z} \sim 15$ (in redshift slices of $\Delta \mathrm{z}=1$ ) down to our limiting magnitude of $\mathrm{m}_{\mathrm{AB}}=28$, compared with at most a few tens of galaxies at this brightness for JWST (Fig. 3) and virtually none such sources from the Euclid and Roman Space observatory missions. Several tens of thousands of $\mathrm{z} \sim 11$ and $\mathrm{z} \sim 12$ ultra-bright galaxies are also predicted for the SPACE mission, dwarfing what are likely to be limited samples with the roman space telescope and Euclid as well as potential follow-up campaigns with JWST (Fig. 3). These numbers are uncertain given the current weak constraints on the luminosity function at high redshift, hence the need for a large SPACE sample to accurately determine the evolution of the LF. If the bright end evolves less rapidly than in these models, these predicted numbers will be lower limits.

The large samples of $\mathrm{z}=11-15$ galaxies from the SPACE mission will be enormously valuable, allowing for a wide variety of follow-up endeavors (spectroscopy from Extremely Large Telescopes (ELTs), X-ray observations with Athena and radio/mm with SKA and ALMA). A recent work detected a candidate for the most remote object in the Universe, at $z=11.1$ [63]. This galaxy is remarkably, and unexpectedly, luminous for a galaxy at such an early time but also very rare. If confirmed, this result implies that the best strategy to detect $\mathrm{z}>10$ is wide fields, as featured by SPACE. With these samples, SPACE will provide the community with new constraints on the prevalence of ultra-bright $\mathrm{z} \sim 13-15$ galaxies and more robust constraints at $\mathrm{z} \sim 11-12$. This can be used to assess the general form of the UV 


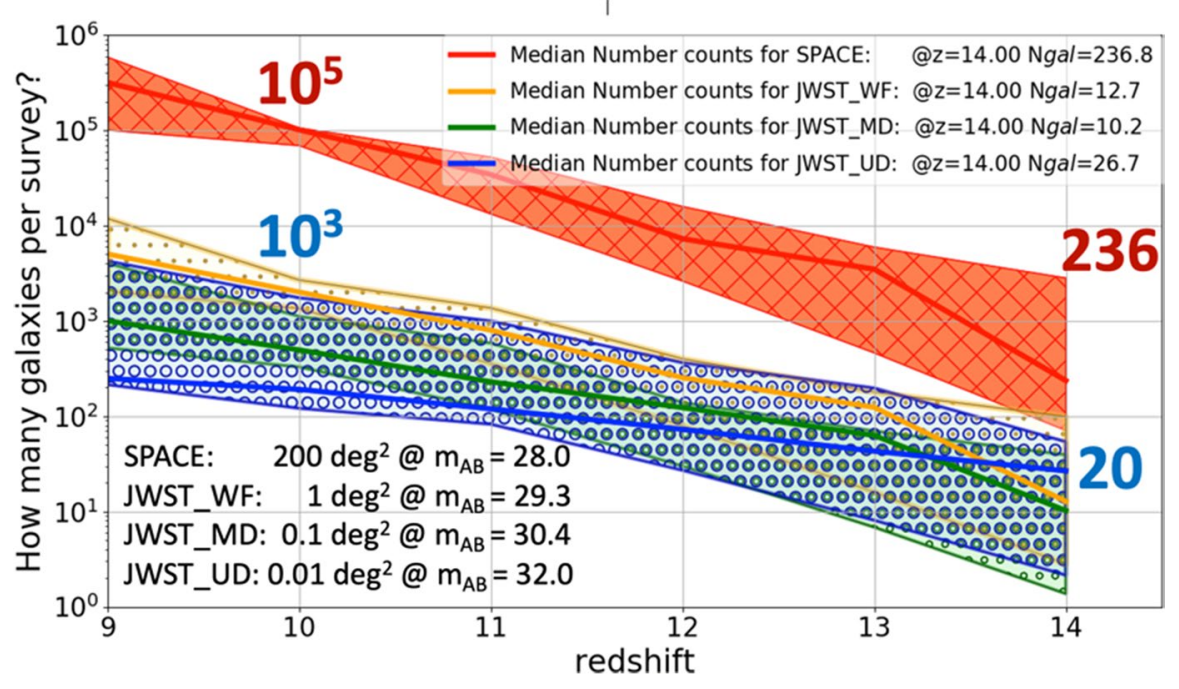

Fig. 3 Number of galaxies to $\mathrm{z}=14$, detected in SPACE 200deg ${ }^{2}$ and the three JWST surveys over $1 \mathrm{deg}^{2}, 0.1 \mathrm{deg}^{2}$ and $0.01 \mathrm{deg}^{2}$ (as defined in [56]). The combined depth / area gives about the same number of objects for each of the JWST surveys. With the same assumptions, SPACE will provide about 240 objects while JWST surveys would have about 20 each. This is a huge gain to understand these galaxies in a statistical way

luminosity function at $\mathrm{z}>10$. There is tentative evidence based on recent observations for the galaxy LF becoming a double power-law shape at $\mathrm{z}>6$, compared with a Schechter function with an exponential cut-off at redshifts lower than this [12]. One possible explanation for this is that the most UV luminous galaxies probed at the highest redshifts actually have lower stellar masses than the same UV luminosity galaxies at lower redshifts, due to both dust extinction [9] and AGN feedback being lower at the high redshifts. This might result in the bright end of the UV LF at higher redshifts increasingly resembling the mass function (a power law) rather than a Schechter function.

While such issues will almost certainly be resolved at $\mathrm{z}<10$ by JWST, Euclid, and the Roman Space Telescope, galaxy formation at $\mathrm{z}=11-15$ faces several unique challenges, the largest of which is the short time scale available for the build-up of the stellar population in a galaxy at such high redshifts. At these redshifts, there are only 100 million years (Myr) available from the formation of the first star to that galaxy forming tens of millions of stars per Myr. This is a very short time scale, particularly in relation to the time scale on which various physical processes operate, i.e., metal enrichment, feedback from supernovae, etc., and it is possible that galaxy formation proceeds differently in this regime. SPACE observations at these very high redshifts are crucial to constrain this.

The unique samples of ultra-bright $\mathrm{z}=11-15$ sources discovered by SPACE will have utility that goes well beyond just studies of their prevalence, as they are extremely high value follow-up targets. Targeting very high redshift galaxies from SPACE with NIR imagers on the next generation of Extremely Large Telescopes 
will give us a direct view of star formation in the most significant mass reservoirs in the early Universe, measuring their size and structural properties. Ultra-bright galaxies out to $\mathrm{z} \sim 7$ have been shown to have a complex, multi-component morphology suggesting merging activity, and it would be extremely interesting to know if such phenomena would also be present in ultra-bright $\mathrm{z} \sim 11-15$ galaxies building up in $\lesssim 100$ Myr. There are fascinating insights to be gained about the dark-matter - galaxy connection at the bright end through studying clustering. These bright galaxies are likely to be the sites of the highest over density peaks in the early Universe, and thus expected to be clustered. SPACE may find protoclusters, the earliest large-scale structures to form.

Follow-up spectroscopy of very high redshift galaxies only discoverable by SPACE (including with the SPACE integral field spectrograph (a multi-object spectrograph can also be considered) and from NIR spectrographs on ELTs) will enable us to determine the physical state of ionized gas (the gas-phase metallicity, electron density, and ionizing flux). This is critical, since very little is known about the physical conditions of star formation in the early Universe. While huge progress will be made in probing these conditions in lower luminosity galaxies with JWST, JWST will likely not look at many ultra-bright galaxies at $\mathrm{z}>12$ with $\mathrm{SFR}_{\mathrm{UV}}>$ a few tens $\mathrm{M}_{\odot} \mathrm{yr}^{-1}$ due to the challenge in finding them prior to SPACE.

\subsubsection{The birth of metals}

A key probe of galaxy evolution is the mass-metallicity relation (e.g. [85]), which indicates how star formation and chemical enrichment proceed in galaxies as a function of mass. If the characteristic timescale (or efficiency) of chemical enrichment depends on the stellar mass, then one would expect the shape (slope and offset) of the mass-metallicity relation to change with redshift. Good determinations have been made at low redshift thanks to the SDSS (e.g. [88]) and more recent work at intermediate redshifts (e.g. [96] at $\mathrm{z} \sim 1$ from DEEP2 on Keck). A small number of galaxies at $\mathrm{z} \sim 3$ also have metallicity estimates (e.g. AMAZE on VLT, [52]). At the higher redshifts there appear to be significant differences between the current small number of observations and theoretical predictions from chemical evolution models (e.g. [25, 84]). It is important to improve the observations (in both number and the mass range covered) and to extend the samples to higher redshifts. SPACE will achieve these observations and be critical in constraining galaxy evolution models (in particular feedback).

The fine structure lines in the mid-IR and far-IR are very powerful to measure the metallicity of massive and high-metallicity galaxies (e.g., [33]). They do not suffer from the dust extinction and from the degeneracy observed for high-metallicity galaxies when using rest-frame optical lines. However, galaxies are expected to have lower masses and low metallicities at $\mathrm{z}>5$ (e.g. [17, 61, 87]). If we want to focus on the rise of metals in the Universe at $z>5$, these IR fine structure lines are not good tracers for low-metallicity galaxies. This is a direct implication of the galaxy formation process in the $\Lambda \mathrm{CDM}$ cosmology (Fig. 4): 
Fig. 4 Even though exceptions exist, the left panels (adapted from [87]) shows that most galaxies at $\mathrm{z}>5$ are expected to be low-mass galaxies at low metallicity (yellow-shaded area). In this metallicity range, the IR fine structure lines reach a plateau and are not sensitive to any change in metallicity as shown in the right panel adapted from Fernández-Ontiveros et al. [33]. Credit: Left: P. Torrey / University of Florida and Right: J.M. Fernandez-Ontiveros / Instituto de Astrofisica de Canarias
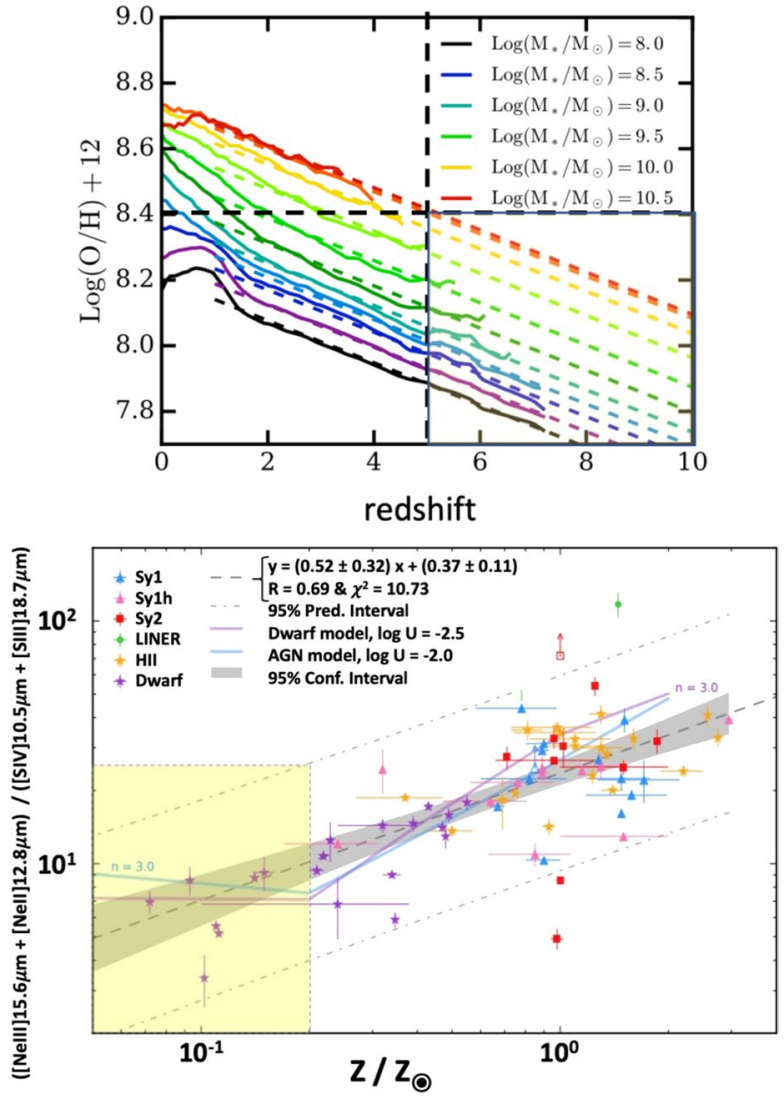

- Galaxies build stellar mass with time

- Higher redshift galaxies have lower mass $\left(\mathrm{M}<10^{10.5} \mathrm{M}_{\odot}\right)$

- We know there is a mass - metallicity relation

- Lower mass galaxies have lower metallicities $\left(\mathrm{Z}<0.2 \mathrm{Z}_{\odot}\right.$ or $12+\log (\mathrm{O} / \mathrm{H})<$ 8.4)

- Higher redshift galaxies have lower metallicities

If we cannot use the fine structure line, what are the options? We can utilize the strong power of the bright rest-frame optical lines and (tentatively) make use of the $3.3 \mu \mathrm{m}$ poly-aromatic hydrocarbon $(\mathrm{PAH})$ feature:

a. There is a large diversity of metallicity indicators (see [53] and Fig. 5). But, traditionally, emission line diagnostics have been used at intermediate redshifts to determine the metallicity of the star-forming gas. For many years a widely-used indicator for the $\mathrm{O} / \mathrm{H}$ abundance has been "R23" [66], which uses [OII], [OIII] and $\mathrm{H} \beta$ as lines which are reasonably close in wavelength (and hence minimizes the effect of differential dust extinction) and accounts to first order for ionization 


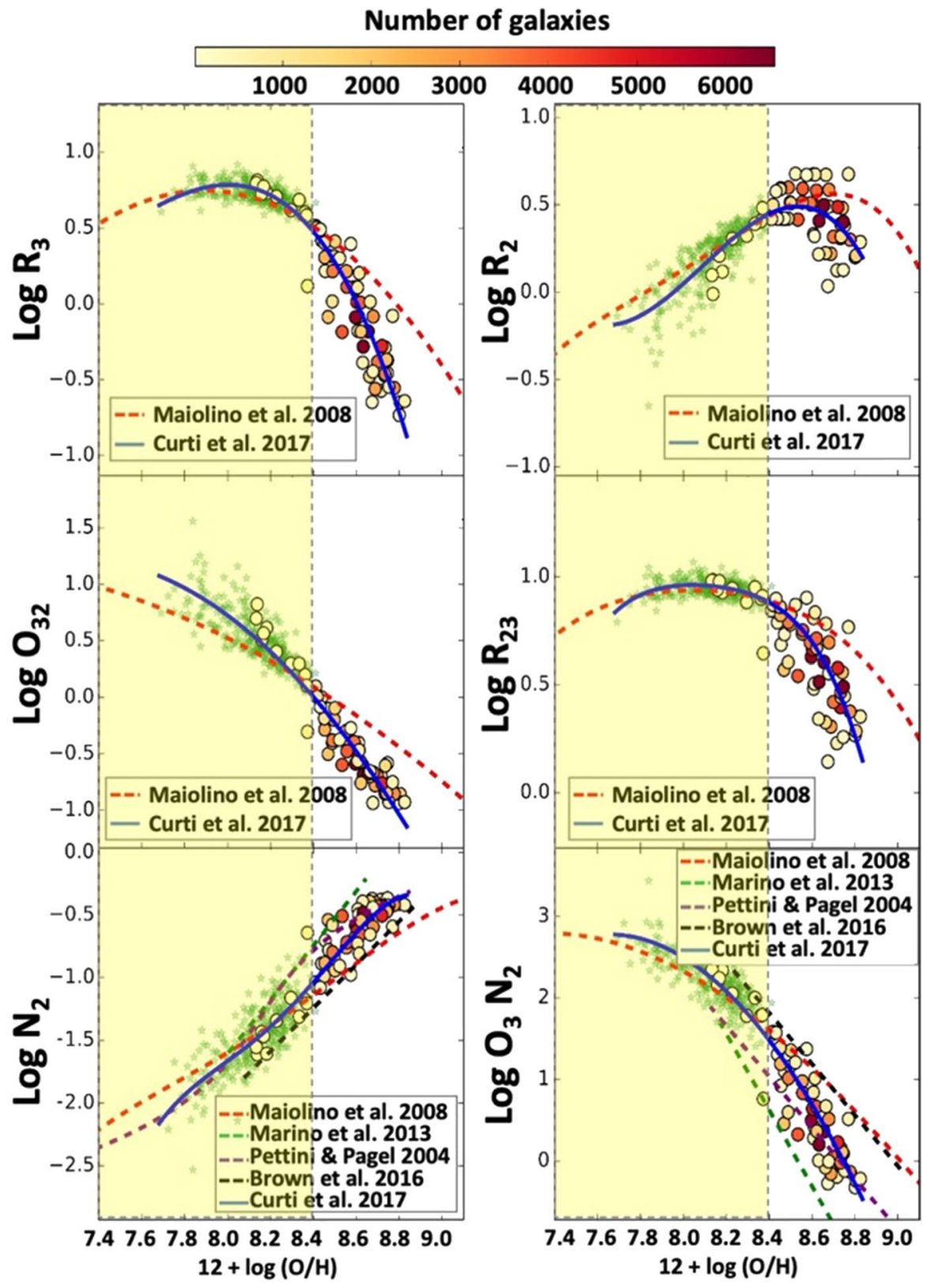

Fig. 5 This plot adapted from Curti et al. [24] shows the diagnosis using strong optical lines as a function of the oxygen abundance. Using these lines, a mid-IR spectrograph would be able to measure the metallicity of galaxies with the same tracer from $\mathrm{z}=0$ to $\mathrm{z}=10$. Some of the tracers show degeneracies while others, involving $\mathrm{H} \alpha$, do not. The preferred tracers for the low-mass, low-metallicity galaxies (yellow region) would be $\mathrm{O} 32$ ([OIII] $\lambda 5007 /[\mathrm{OII}] \lambda 3727), \mathrm{N} 2([\mathrm{NII}] \lambda 6584 / \mathrm{H} \alpha)$ and O3N2 ([OIII] $\lambda 5007 / \mathrm{H} \beta) /$ ([NII] $\lambda 6584 / \mathrm{H} \alpha)$. This means that we need to be able to observe enough galaxies (mapping efficiency) in the good wavelength range, accounting for the redshift. Credit: M. Curti, G. Cresci \& F. Mannucci, INAF 
by using two species of oxygen (see also the updated $\mathrm{O} / \mathrm{H}$ method of [24]). We can determine R23 out to $\mathrm{z} \sim 9$ with SPACE. However, there is a well-known "double fork" in the plot of R23 against metallicity, but the [NII]/H $\alpha$ line ratio can be used to break this degeneracy and determine if a galaxy lies on the upper or

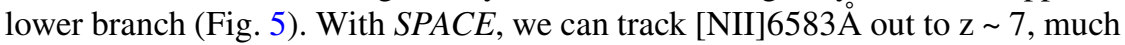
further than the current $\mathrm{z} \sim 2.5$ limit. Our SPACE spectroscopy will access several emission lines, enabling us to use "BPT" diagrams [4] to address the nature of the photoionization in individual galaxies (i.e. star formation vs. AGN). The line ratios will also probe the ionization parameter, and recent work has suggested that this rises with redshift ([44] at $\mathrm{z} \sim 0.6)$.

b. We will also be sensitive to the PAH features in galaxies at high redshift. The PAH are destroyed in low-Z environment by the UV radiation field, which propagates more easily due to the lower dust content for galaxies with lower metallicities [10, $36,38]$. The PAH3.3 $\mu \mathrm{m}$ could be an excellent vector to measure the metallicity over a very wide range of redshifts, using the same indicator. The low thermal background of SPACE permits much deeper observation in the MIR at $\lambda>13 \mu \mathrm{m}$ than JWST-MIRI. In this range, we observe a strong and linear decrease of $\mathrm{q}_{\mathrm{PAH}}$, the mass fraction of PAH (Fig. 6) that seems to be correlated to the metallicity of the observed galaxies. Several interpretations for this effect exist. But the leading one is that PAH are destroyed in low-Z environment by the UV radiation field, which propagates more easily due to the lower dust content for galaxies with lower metallicities $[10,38,50]$. The PAH3.3 $\mu \mathrm{m}$ will be an excellent vector to measure the metallicity over a very wide range of redshifts, using the same metallicity indicator.

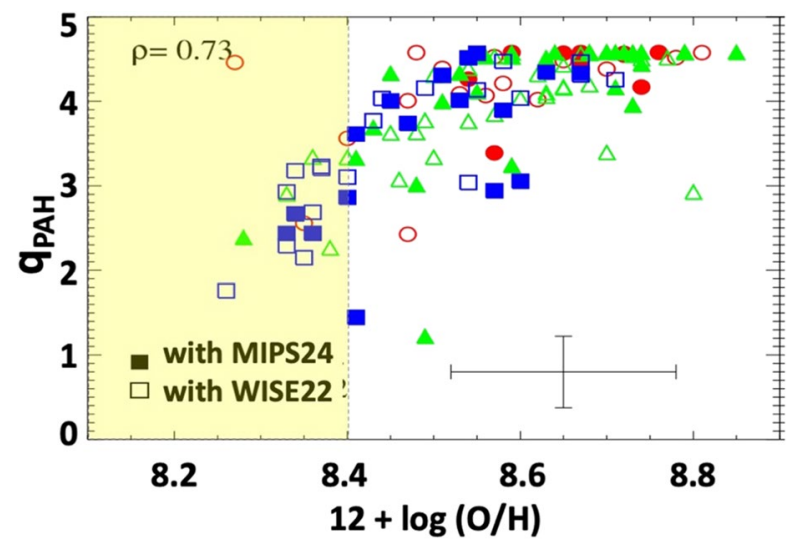

Fig. 6 This plot, extracted from Ciesla et al. [22] suggests that PAH are destroyed by the UV radiation field in the specific metallic environment of low-metallicity galaxies in the local Universe and probably also in the early Universe. Following up the PAH3.3 $\mu \mathrm{m}$ feature with SPACE will allow to better understand this behavior and to apply it to very high-redshift galaxies to measure the metallicity of these objects, with the goal of charting the rise of metals in the early times. Credit: L. Ciesla / LAM 
Of course, there is not just one "number" which describes the chemical enrichment of an individual galaxy. We can improve on R23 metallicity by measuring abundances of different elements. This would provide strong constraints on galaxy evolution, and in particular the relative contribution to chemical enrichment from core-collapse supernovae ( $\mathrm{SNe}$ ) and asymptotic giant branch (AGB) stars (e.g. [62]), which have different timescales and hence the abundance patterns should evolve strongly with redshift. The N/O ratio can be determined using the [SII], [NII], H $\beta$ and [OIII] lines to constrain the ionization parameter [28], and similarly the CIII]1909 and CIV lines provide the carbon abundance [2]. Among AGB stars, nitrogen is produced from relatively massive stars $\left(\sim 5 \mathrm{M}_{\odot}\right)$, while carbon is produced by the low-mass end $\left(1-3 \mathrm{M}_{\odot}\right)$ with longer lifetimes. Oxygen is produced very promptly from core-collapse $\mathrm{SNae}$ (with $>8 \mathrm{M}_{\odot}$ progenitors). The relative abundances probed by SPACE spectroscopy tell us about the galactic archeology, and potentially any evolution of the stellar initial mass function. For the brighter sources at the medium spectral resolution $(\mathrm{R}=600-1000)$ we may be able to detect absorption lines - this may constrain the stellar metallicity (rather than gas phase) and be less affected by the ionization parameter and dust. However, many of the absorption lines arise from the interstellar medium (ISM) rather than from stars. This gives information on the velocity of outflows (the feedback from star formation).

Metal enrichment and supernovae at high redshift Probing the metallicity in ultrabright galaxies will be key to understanding their evolution, as it could provide us with the earliest high-quality information about the chemical enrichment occurring in the most massive reservoirs of star formation in the early Universe. Such probes of the metallicity are likely to be very interesting as they could provide us with information on the nature of some of the first SNe that occur in the Universe and enrich the interstellar medium. This subject has huge potential, as it is thought that many of the first stars, being metal free and massive (with masses of the order of $>40 \mathrm{M}_{\odot}$, given the limited cooling of molecular hydrogen e.g., [13]), undergo pair-instability supernovae (PISNe). In this regard, ultra-bright $\mathrm{z} \sim 14-15$ galaxies - due to both their brightness and very young ages $(<100 \mathrm{Myr})$ - arguably provide us some of the best targets to look for evidence of enrichment from such $\mathrm{SNe}$ in the early Universe.

By observing fields repeatedly, SPACE may also be able to directly detect these SNe. PISNe produce huge amounts of iron (from Nickel-56) with a long plateau, and thus can be detectable even at high redshifts. The cosmic rate of PISNe depends on the formation rate of stars in the mass range $40-300 \mathrm{M}_{\odot}$. In other words, the detection or non-detection of PISNe can constrain theoretical models. Whalen et al. [92] estimate magnitudes for PISNe at $\mathrm{z}=20-30$, which can rise as bright as $\mathrm{m}_{\mathrm{AB}}=$ $26 \mathrm{mag}$ at $2 \mu \mathrm{m}$ and $\mathrm{m}_{\mathrm{AB}}=27 \mathrm{mag}$ at $4.4 \mu \mathrm{m}$. These are within reach of the proposed SPACE survey to $\mathrm{m}_{\mathrm{AB}}=28$, and hence SPACE has both the sensitivity and the large area mapping capability to search for PISNe. JWST will lack the field of view to make detecting this population a realistic proposition, and the Rubin Observatory will be limited in sensitivity at $2 \mu \mathrm{m}$ and beyond, limiting its redshift range. The light curve decline will be very long due to the time dilation at these redshifts, and we 
will adapt our survey strategy to re-visit target fields with the appropriate cadence (spanning months and years).

\subsubsection{The assembly of stellar mass in galaxies}

Parallel to the quest for the highest redshift star-forming galaxies, the SPACE imaging survey will also detect the redshifted starlight from the most massive galaxies over a wide range of redshifts $(4<\mathrm{z}<10)$, where the wavelength coverage of SPACE extends to the rest-frame optical. The presence of massive galaxies, i.e. $\mathbf{M}_{\text {star }}>5 \times 10^{10} \mathrm{M}_{\odot}$, at early cosmic times constitutes a fundamental constraint for galaxy formation models. According to these models, no such massive galaxies are expected to have formed at $\mathrm{z}>6$, when the Universe was less than a billion years old and should become common only much later in cosmic time. This is a consequence of our current understanding of how galaxy formation proceeds in the $\Lambda \mathrm{CDM}$ cosmological model. However, different observational results are starting to challenge these predictions with the discovery of a few rare massive galaxy candidates at high redshift (e.g. [20, 29, 82]). There is some evidence that the stellar mass - halo mass relation evolves, such that star formation is more efficient at high redshift $[7,34,40]$. But the samples are very small, and clearly much larger samples are required. This will provide crucial insights into the feedback mechanisms which regulate the assembly of stellar mass in dark matter haloes.

Given the rarity of these objects, deriving a useful constraint for galaxy formation models requires the analysis of deep galaxy surveys over wide areas of the sky covering at least several tens of square degrees. No current or forthcoming telescope can provide deep IR images over such large areas. SPACE will be the only telescope able to quantify the presence of massive galaxies at early cosmic times, resulting in crucial constraints for galaxy formation theories. SPACE will provide excellent stellar masses for huge numbers of objects in the fields, over much larger areas than JWST will be able to address. Indeed, by extrapolating the results of current galaxy surveys over small areas, we expect SPACE to find around 30,000 massive galaxies $\left(>10^{10}\right.$ $\mathrm{M}_{\odot}$ ) in a $\sim 200 \mathrm{deg}^{2}$ survey at $\mathrm{z}=6$ (in a redshift slice of $\Delta \mathrm{z}=1$ ), and potentially 5,000 at $z=7$, along with more than 100,000 massive galaxies at $z \sim 4-5$. These unprecedented statistics will allow us, for the first time, to study galaxy clustering in the early Universe with the same level of detail as we currently do at $\mathrm{z}<1$ [98] and recently started doing at $\mathrm{z} \sim 3$ [31]. SPACE will open up a new era of 'precision cosmology' at high z, only limited by the accuracy of photometric redshifts (which will be self-calibrated with the thousands of spectroscopic redshifts that SPACE itself will provide). There are no current constraints on the number of massive galaxies at higher redshift, but if we assume the same shape of the galaxy stellar mass function as at lower redshift, we would expect SPACE to find 1,500 massive galaxies at $\mathrm{z} \sim 8$ $\left(>10^{10} \mathrm{M}_{\odot}\right.$ and $\left.\Delta \mathrm{z}=1\right)$ and 50-100 at $\mathrm{z} \sim 9-10$. The distinctive wavelength coverage of SPACE $(1-28 \mu \mathrm{m})$, which goes beyond that of any other planned wide-field facility, is uniquely able to determine the stellar masses of all these high-z galaxies (Fig. 7). The information on $\mathrm{M}_{\text {star }}$ will provide a unique opportunity to study the growth of baryonic structures from the early Universe to the present day. 


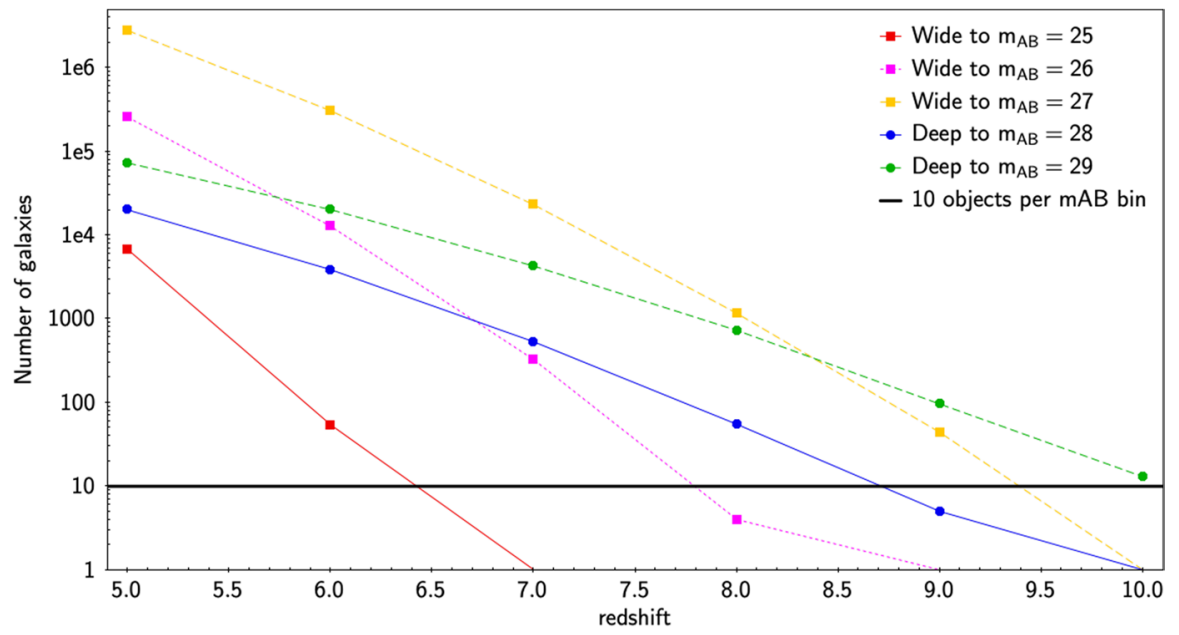

Fig. 7 Number of galaxies that will be detected by the two Roman Space Telescope (formerly WFIRST) Deep and Wide surveys at $5<\mathrm{z}<10$. SPACE will be able to estimate $\mathrm{M}_{\star}$ for a representative sample of at least 10 galaxies in the bins where the Roman Space Telescope detected objects to $\mathrm{z}=10$. The Roman Space Telescope will provide the SFR from the rest-frame FUV

More specifically, SPACE will provide a unique way of following-up the Roman Space Telescope Deep and WFIRST-Wide surveys. After the Roman Space Telescope, no projects will be able to measure the star formation rate (SFR), the stellar mass $\left(M_{\star}\right)$, and the dust attenuation of galaxies detected from the Roman Space Telescope surveys until we have SPACE photometric capabilities. The rest-frame UV spectrum will provide an access to young stars that are likely to be predominant at $\mathrm{z}$ $>5$ but, if we want to perform a complete census, including potential older stars, we need the rest-frame optical and near-IR. To follow up the Roman Space Telescope's objects at $\mathrm{z}>5$, after JWST, we need a wide-field NIR+MIR instrument From these unique data, we will be able to measure the evolution of the mass function (cosmic mass assembly), the SFR density, and average dust attenuation for a representative sample of galaxies at $5<\mathrm{z}<10$. In 2035+, a number of facilities will have surveyed the sky to identify galaxies before and slightly after the end of reionization. JWST will certainly open up this era but galaxies are expected to be very rare and faint at $5<\mathrm{z}<10$. the Roman Space Telescope's Deep and Wide surveys will provide a unique opportunity to build statistical samples (Table 1).

\subsubsection{Formation and growth of the first supermassive black holes}

All massive galaxies today appear to harbor a central supermassive black hole. A key question is how the first massive black holes form and grow? This can be addressed by searching for Active Galactic Nuclei (AGN) at high redshift. The most massive black holes at $\mathrm{z} \sim 6-7$ are $\sim 10^{9}-10^{10} \mathrm{M}_{\odot}$ which already places stringent constraints on their formation mechanism and their rate of growth from the first black-hole seeds to just $\mathrm{z} \sim 6-7$. This will be achieved from SPACE by providing 
Table 1 Summary of the requirements for the main near-IR + mid-IR instrument

\begin{tabular}{|c|c|}
\hline Size of the primary mirror & $4-6 m$ \\
\hline Wavelength range & $3-28 \mu \mathrm{m}$ \\
\hline Pixel scale imaging & $0.2 \operatorname{arcsec}$ \\
\hline Spectral resolution imaging & $3-5$ \\
\hline Instantaneous imaging field of view & $0.5-1$ sq. deg. \\
\hline Limiting flux mAB (1h, @ 5 $\mu \mathrm{m}, \mathrm{SNR}=5)$ & $28-29$ \\
\hline Pixel scale spectroscopy & $0.4 \operatorname{arcsec}$ \\
\hline Instantaneous Spectroscopic field of view (MOS) & $0.5-1$ sq. deg. \\
\hline Instantaneous Spectroscopic field of view (IFS) & 1 sq. arcmin. \\
\hline Spectral resolution (Low resolution) & $20-50$ \\
\hline Spectral resolution (Medium resolution) & $500-1000$ \\
\hline Limiting line flux (1h, @ $5 \mu \mathrm{m}$ SNR = 5) & $10^{-18}-10^{-19} \mathrm{erg} / \mathrm{cm} 2 / \mathrm{s}$ \\
\hline
\end{tabular}

measurements of the quasar luminosity function out to $\mathrm{z} \sim 10-12$ for luminosities $\sim 5$ magnitudes below the absolute magnitude $\mathrm{M}^{*}$ through photometric identification with broad band colours.

Spectroscopic follow-up of these candidate quasars (potentially with the SPACE integral field or multi-object spectrograph) will measure the black-hole accretion properties, such as the Eddington ratio (using virial estimators from broad emissionline components), as well as the metal enrichment from the strength of metal lines. A puzzling fact about the highest-redshift quasars currently known is that they are all universally metal rich, suggesting that they have already undergone a substantial amount of evolution. Spectroscopy of quasars from SPACE at even higher redshifts and fainter magnitudes will address whether this persists out to yet higher redshifts and for lower-luminosity systems. A drop in metal richness will have implications for the fraction of obscured systems since the obscuration is dominated by metals and we may therefore expect an increasing population of dust-free and unobscured quasars at very high redshift. SPACE imaging of fields surveyed by Athena (for $\mathrm{X}$-ray sources) and SKA (for radio sources) will determine the fraction of obscured/ unobscured systems. These properties may be connected to the host-galaxy environment and the large-scale environment within which quasars reside, and the largescale quasar environment can be measured using the large SPACE IFU through the identification of associated galaxies and AGN with the quasar. The predictions shown in Fig. 8 suggest that SPACE will detect in a $200 \mathrm{deg}^{2}$ survey a few hundred quasars at $\mathrm{z}>6$, up to $\sim 100$ at $\mathrm{z}>8$, and $\sim 5-10$ at $\mathrm{z}>10$. We note that there are currently only a handful of QSOs known at $z>7$ (e.g., [5, 59]).

We also note that in a $200 \mathrm{deg}^{2}$ survey with Athena, the predicted numbers of $\mathrm{z}>8$ and $\mathrm{z}>10$ AGN with an X-ray luminosity $\mathrm{L}_{\mathrm{X}}>10^{43} \mathrm{erg} / \mathrm{s}$ (broadly equivalent to the rest-frame $1450 \AA$ depth of $S P A C E$ ), are similar to those of $S P A C E$. Although we would expect significant overlap in the identified source populations between Athena and SPACE, they will also be very complementary, with Athena being more effective at detecting the obscured systems but unable to measure redshifts (and hence source properties) for the majority, which 


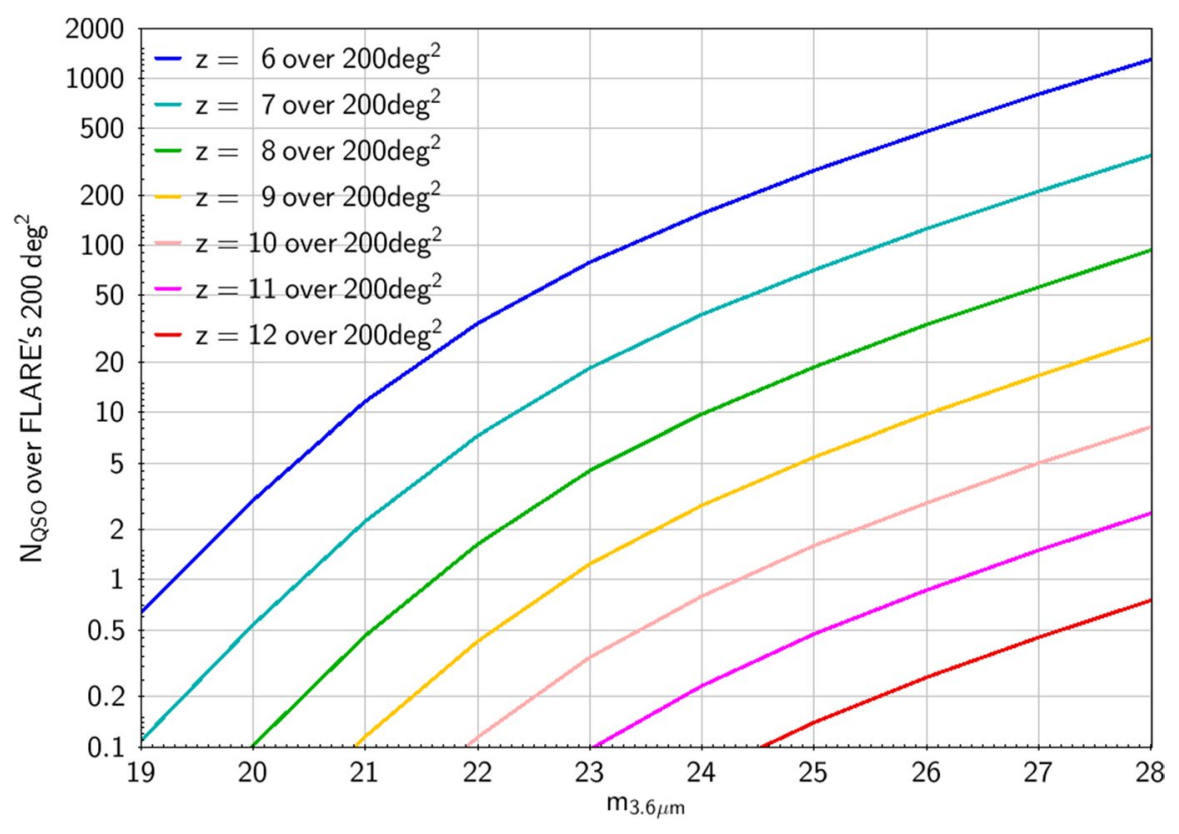

Fig. 8 Extracted from Burgarella et al. [18]. Basic predictions calculated by David Rosario using the Venemans et al. [89] quasar luminosity function and the SDSS composite quasar spectrum for a $200 \mathrm{deg}^{2}$ survey. SPACE limiting magnitude will be $\mathrm{m}_{\mathrm{AB}}=28$

will require SPACE. The timescale of Athena is well suited to follow-up SPACE detections, and, as well as AGN Athena could potentially target clusters of galaxies at $\mathrm{z}>2$ discovered by SPACE to measure the temperature of the intracluster medium (and hence the total cluster mass). Note that SPACE will also feature a wide survey to study the Milky Way. Although this survey will suffer from high Milky way extinctions since it is, by design, close to the disc of our Galaxy, it might provide an additional sample of bright quasars.

Deep, broadband, wide-field photometric surveys such as the one presented in Section 2.2.1, provide a wealth of information on galaxies. However, this photometric information is not sufficient to gather a complete view of the formation and evolution of galaxies. Some physical information, such as the kinematic state of the galaxies and their chemical abundances, cannot readily be extracted from photometric data: spectroscopic observations are required. Furthermore, while continuum-bright galaxies can easily be detected using broad bands, part of the galaxy population is missed by these surveys: spectroscopic surveys without any prior selection do not demonstrate the same redshift distribution. Even spectroscopic follow-up of imaging surveys cannot provide a complete census of star-forming galaxies as some galaxies with extremely high equivalent width emission lines are undetected in broad-band imaging even with the deepest facilities (as shown by [3]). 


\subsubsection{A wide-area integral field spectroscopic survey with SPACE: emission lines from high redshift star forming galaxies}

The SPACE IFU will allow for the first time an unbiased deep survey for emission line sources over an area of $\sim 1.5 \mathrm{deg}^{2}$ in total, many orders of magnitude larger than will be surveyed with the JWST NIRSpec IFU. Thanks to the NIR+MIR coverage of SPACE $(1-28 \mu \mathrm{m})$ and when they are bright enough, $\mathrm{H} \alpha$ and the other optical lines can be traced out to the first galaxies. The well-known rest-UV lines of Ly $\alpha$, HeII1640, OIII]1663, CIII]1909, and CIV1550 can be observed for high-redshift sources $(z>5-7)$ and up to the largest distances where no galaxies have yet been found.

\subsubsection{An emission line survey at $z>7$}

Optical emission lines provide invaluable information on the chemical composition, ISM properties, nature/hardness of the ionizing source, and on the instantaneous SFR, stellar age, and related properties. But to collect a large number of objects needs wide and deep spectroscopic observations that are extremely difficult, even for JWST [26, 93]. For sources with magnitudes brighter than $\mathrm{m}_{\mathrm{AB}}=28$ (detected in the continuum survey), the IFU survey will detect [OIII]5007 emitters with rest-

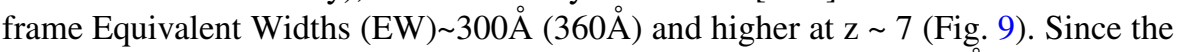
typical strength of $[\mathrm{OIII}]$ in star-forming galaxies at high-z is $\sim 1000 \AA$ according to
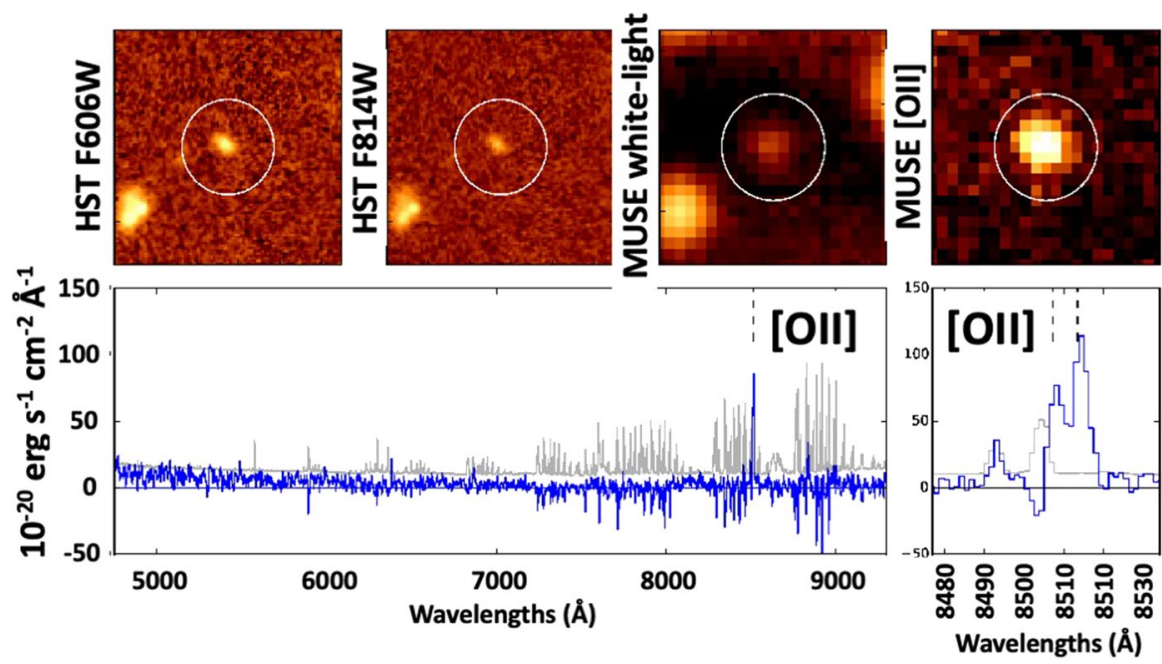

Fig. 9 Integral-field spectroscopic observations with MUSE on the VLT [3] in the Hubble Deep Field South showed that as many as 30\% of entire Ly $\alpha$ emitter samples have no HST counterparts with I814 $>29.5$. For instance, for this $z=5.08$ object, HST broad bands are shown (top left), the MUSE reconstructed white-light and Ly $\alpha$ narrow band images (top right). The 1-arcsec radius circles show the emission line location. The full spectrum (in blue), smoothed with a $4 \AA$ boxcar, and its $3 \sigma$ error (in grey) are displayed below. A zoom of the unsmoothed spectrum, centered around the Ly $\alpha$ emission line, is shown (bottom right). Credit: R. Bacon / CRAL - Observatoire de Lyon 
current estimates [45, 75], SPACE should detect this line for all sources to the depth of the continuum survey $\left(\mathrm{m}_{\mathrm{AB}}=28\right.$ for the SPACE imaging), corresponding to several hundred sources with [OIII] at $\mathrm{z} \sim 7$ (Fig. 10). Furthermore, since fainter, less massive galaxies generally have stronger emission lines, the IFU survey should discover pure emission lines sources undetected in the continuum surveys. This point is illustrated in Fig. 9 where a Ly $\alpha$ galaxy at $z=5.08$ does not show any HST counterparts in the broad F606W and F814W bands. As many as 30\% of entire Ly $\alpha$ emitter samples have no HST counterparts with I814W $>29.5$.

The rest-UV emission lines of star-forming galaxies have lower equivalent widths than the optical lines. The strongest ones are Ly $\alpha$ (up to $\sim 200-300 \AA$ for normal populations and higher for "exotic" cases, e.g. Pop III) and CIII]1909 with EW up

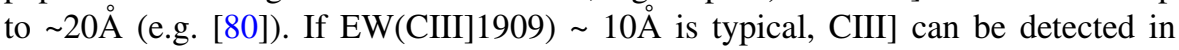
fairly bright galaxies $\left(\mathrm{m}_{\mathrm{AB}} \sim 26-26.5\right.$ or brighter) at $\mathrm{z} \sim 7$ to 9 with SPACE, and all the sources whose UV lines will be detected in the spectroscopic IFU survey (down to fluxes $\sim 10^{-18} \mathrm{erg} / \mathrm{cm}^{2} / \mathrm{s}$ ) will also be detected in the continuum survey. For these sources the line detection will provide spectroscopic redshifts and a key insight into the physical properties of galaxies including the hardness of the radiation field, chemical composition, ionizing photon production and others, as shown e.g. by recent pilot studies using ground-based near-IR spectroscopy $[79,80]$ at $\mathrm{z} \sim$ 7 and recent models (e.g. $[32,60])$.

\subsubsection{The evolution of the star formation rate density at $z=2-7$}

Selecting on $\mathrm{H} \alpha$ emission has been shown to be by far the most complete way to select star-forming galaxies. Due to its redder wavelength and typically high luminosity, selecting galaxies by their $\mathrm{H} \alpha$ emission can efficiently recover a large range of star-forming galaxies, from very blue to the most dusty/sub-mm galaxies (e.g. [64]). Furthermore, the $\mathrm{H} \alpha$ emission line may turn out to be even more important

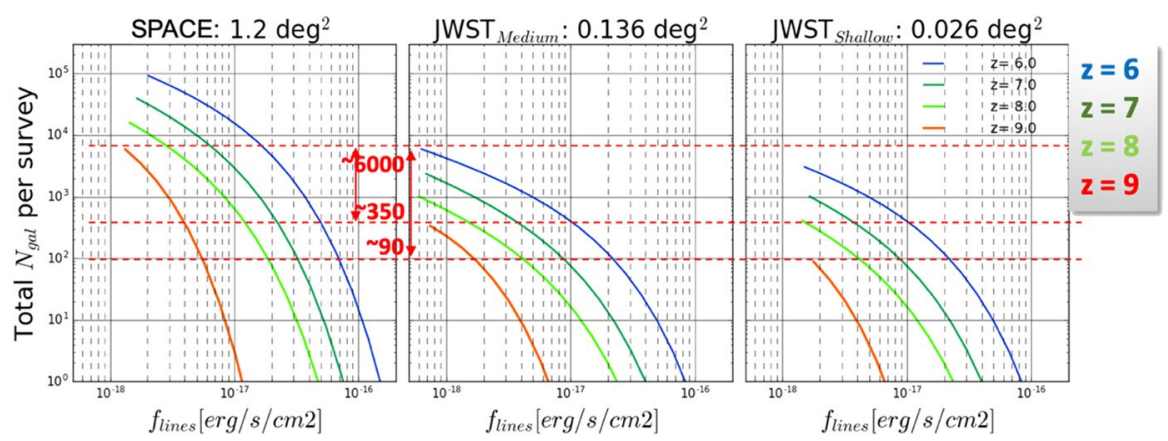

Fig. 10 Using UV luminosity functions, we can estimate the number of galaxies that could be detectable by SPACE and by the JWST surveys (R $>1000$ ). The JWST Deep Spectroscopic survey is not plotted here because it is not in the same range. For the medium and deep spectroscopic surveys, we present the number of galaxies detected via their [OIII]5007 line (the same ratio is found for H-alpha and [OII]3727 but the redshift range is linked to the rest-frame wavelength of the line). At any redshift, SPACE will detect more than 10 times the number of objects that JWST could collect 
as a star formation indicator at higher redshift if star formation histories are significantly bursty.

SPACE will be the first mission to measure the $\mathrm{H} \alpha$ luminosity function (Fig. 10), and its evolution beyond $\mathrm{z} 2$ and out to $\mathrm{z} \sim 6-7$. Most importantly, the spectroscopic/ IFU capabilities mean that $S P A C E$ will be able to do this on a large volume $(\sim 1.5$ $\left.\operatorname{deg}^{2}\right)$, but also in a completely unbiased way without any pre-selection on galaxies being Lyman-break galaxies. SPACE will provide a unique insight into the overlap of purely $\mathrm{H} \alpha$ selected galaxies up to $\mathrm{z} 7$ and those selected based on imaging and with the Lyman-break technique. How much star-formation are we missing? Are H $\alpha$ and UV star formation rates consistent? Furthermore, these observations will also cover other emission lines, key to understanding the properties of high redshift galaxies. For these unique samples, the IFU will allow SPACE to also obtain spatially resolved information, including line ratios (for metallicity and dust extinction measures) and to obtain the dynamics (and hence masses) of high redshift galaxies.

\subsubsection{Searching for the first generation of stars with SPACE spectroscopy}

While several simulations and predictions have been made regarding the first generation of stars and black holes, including their hosts and redshift distribution (e.g. [71, $78,86,95])$, observational evidence has been lagging and failing to provide direct tests. This is essentially due to the lack of current instrumentation and telescopes to truly test them at high enough redshift. One needs to simultaneously probe high enough redshift, probe large enough volumes, have the sensitivity in the NIR, but also conduct surveys with an inclusive enough selection function such that extreme/ interesting objects are not wrongly classed as stars or low redshift interlopers.

In practice, in order to truly make progress on what are expected to be rare, shortlived sources residing at very high redshifts, one clearly needs: i) near- to mid-IR coverage, ii) imaging and spectroscopic coverage and iii) capacity to cover the large enough volumes/areas that go significantly beyond the current state-of-the-art in an unbiased way. SPACE is the only mission that can combine all the necessary capabilities to make revolutionary discoveries on this end.

Prior to SPACE, JWST will have a very important role in further pushing this and providing the need to further develop tools to understand likely extreme stellar populations. However, during its limited, highly subscribed lifetime, it will mostly conduct spectroscopic follow-up of Lyman-break selected galaxies. While some of those may turn out to be exciting sources, JWST is unlikely to be able to find the rare sources which only SPACE has the large enough volume to find. Furthermore, it is already clear that the search for pristine and/or extreme sources is not going to be simple at all. For example, one cannot simply detect high enough HeII equivalent width and be sure of the pristine nature of the source (e.g. [41, 76]). Regardless, it is also clear that the selection function may be key, and having an unbiased, blind IFU selection function in the key observable window (NIR and MIR) will provide the unprecedented survey capability to uncover tens to hundreds of sources similar to, e.g., CR7 [76].

On the other hand, several studies (e.g. [86, 90]) show that Pop III star formation, and direct collapse black holes $(\mathrm{DCBH})$ form and remain well within the observable 
capabilities of SPACE. However, since we do not know their intrinsic spectra, nor their diversity (and that of their hosts), only a blind spectroscopic survey can truly find and study them (since some may have strange colours which may not fit into the standard broad-band Lyman break selection). SPACE will probe other lines apart from HeII, which is key to unveil the metallicity and physical conditions.

Within SPACE's IFU survey, and with the current estimates for number densities of extreme sources based on either CR7 or e.g. [90] one would expect number densities around $10^{-5}$ to $10^{-6} \mathrm{Mpc}^{-3}$ around $\mathrm{z} 7$ but rising at higher redshift. This means SPACE should be able to detect a truly statistical sample of $\sim 100$ of such sources without any pre-selection and to provide unique constraints on their nature directly by means of all the rest-frame UV lines detected.

\section{Milky way studies: from the global structure to dust characterization}

Stars form from interstellar cloud material. Massive stars form only in giant molecular clouds while solar-mass stars form in both small clouds and giant ones. Herschel has confirmed the prevalence of filaments inside interstellar clouds, along which prestellar cores and protostars could be preferentially seen (Fig. 11 from [83] see also [1];). However, we still do not understand in detail a number of facts such as the role of the galactic environment on the formation and dynamics of molecular

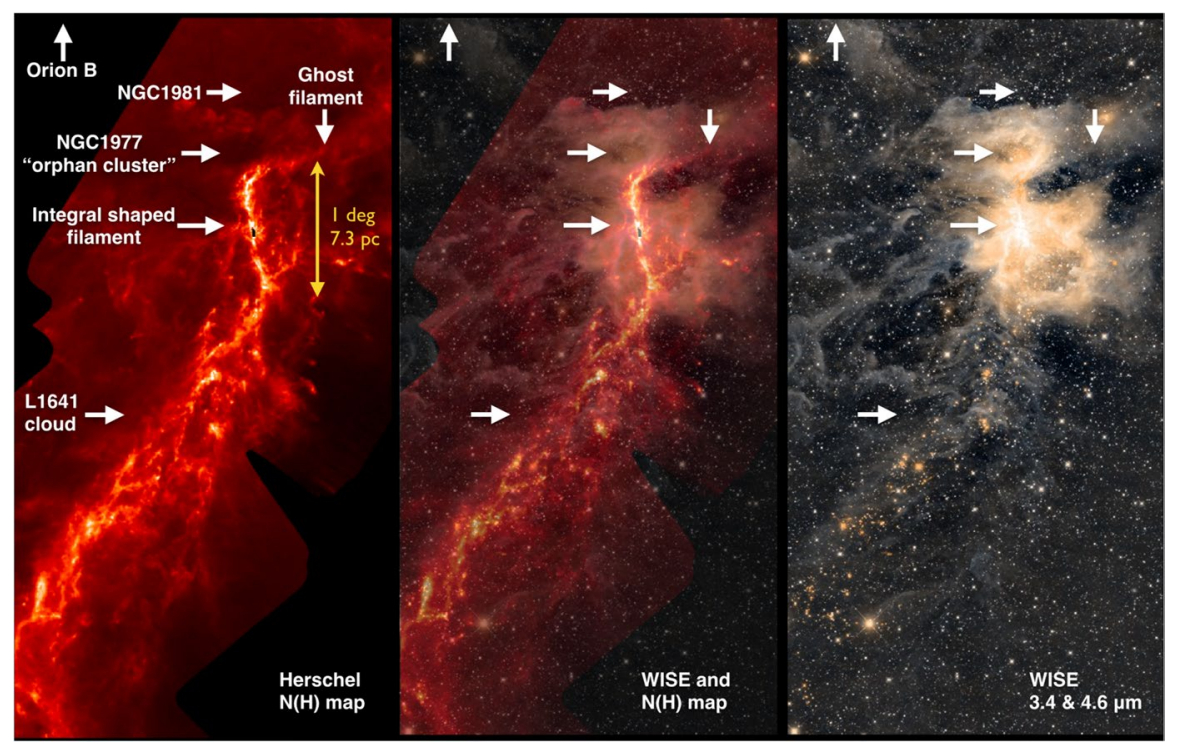

Fig. 11 Extracted from Stutz and Gould [83]. Images of the Orion A star-forming cloud, showing the integral-shaped filament, the two star clusters outside the filament, and the cloud L1641 to the South. Left: density map reconstructed from Herschel data, right: infrared image taken with the WISE space telescope [47], center: combination of the two. Credit: A. M. Stutz / MPIA (now Univ. De Concepcion, Chile) 
clouds ; the interplay between gravity, turbulence, and magnetic field in shaping the prestellar cores from cloud fragmentation; the net effect of star-formation feedback due to supernovae, far-UV fluxes, and stellar winds from young (proto-)stars; the relation between the masses of the clouds, of the cores and of the newborn stars, the formation of planets, the role of dust and ices in the clouds and in the water enrichment of the Earth, etc. Progressing on those open questions requires a multi-scale observational approach to characterize the interactions from the Galactic-structure down to the protostellar-disc scales, revealing dust properties and ice coverage in all clouds. With a large field of view, a reasonably high angular resolution, a near- to far-IR detector, SPACE will provide an opportunity for such multi-scale analysis on statistical grounds.

At large scales, the 3D distribution of the molecular clouds in the Galactic Plane is challenging to infer. It is critical to understand the environmental effect on star formation, such as the impact of spiral arms or of the galactic bar on the molecular cloud formation and evolution. Moreover, distance estimates are essential to derive the cloud masses and identify the associated populations of young stellar objects (YSOs). Although the 3D structure of the ISM has already been explored, most methods rely on too simple assumptions and loosely-constrained ancillary data that limit the distance estimate, like the Galaxy rotation curve for kinematic distances or the dust temperature for inversion of dust emission maps.

The 3D structure of the ISM in the Galaxy will benefit from SPACE using a method free of the gas and dust biases mentioned above. The comparison of the star photometry with a model of stellar population synthesis (such as the so-called Besançon Galaxy model) means that each star can be assigned a distance and a reddening value to each star. The reddening is directly related to the column density which eventually yields a large-scale 3D map of the ISM. Previous attempts to build 3D extinction maps of the Galactic Plane using 2MASS led to 10 arcmin resolution maps for the inner plane. Higher resolution can be achieved by including Gaia [35] data, but only for the first few kiloparsecs, due to the strong extinction in the Galactic disc in the visible domain. Attempts to reach a higher spatial resolution with the deeper IR survey UKIDSS failed because in the near-IR spectral range the giant and dwarf stars cannot be separated by their colour if they are too faint. SPACE with its $3 \mu \mathrm{m}$ to $28 \mu \mathrm{m}$ range and exquisite sensitivity will allow us to separate the two stellar populations and therefore build much better extinction maps. With deep infrared data from $3 \mu \mathrm{m}$ to $28 \mu \mathrm{m}$, it will be possible to improve the spatial resolution to the sub-arcmin scale, reaching the arcsecond scale for clouds seen in absorption against the bright background in the mid-IR range, and probe lines of sight farther than 15 $\mathrm{kpc}$. Such a map will be a goldmine for star formation investigations and more generally for Galactic structure studies.

The study of star-forming regions (SFReg) spans many scales, from the molecular complex scale (a few tens of pc for the most massive ones), to the filament (a few pc), core $(\sim 0.1 \mathrm{pc})$ and, disc $(\sim 0.001 \mathrm{pc})$ scales. It is a key to the understanding of star formation. Indeed, massive SFRegs play a pivotal role in the evolution of the Milky Way, and more generally of disc galaxies, since they are responsible for the formation of most stars in the Galaxy and are the only places where massive stars form. It has been understood early that the evolution of massive 
SFRegs is driven by the complex interplay between gravity, turbulence and magnetic fields, under the ambiguous influence of massive stars. The discovery of the ubiquity of filaments in the interstellar medium (ISM) has made it clear that these structures play a central role in how the gas flows from the diffuse large scales to the dense, star-forming cores. However, a major bottleneck is to disentangle the contributions of each physical process, because gravity and turbulence are multi-scale processes, and because they feed each other. Great progress was achieved in the last decade by numerical simulations, but a real breakthrough can only arise from a statistical comparison with observed SFRegs. However, observations remain hampered by the limited resolution and sensitivity of past and current facilities, which only enable one to fully characterize a handful of massive SFRegs. At $8 \mu \mathrm{m}$, the $4 \mathrm{~m}$ mirror of SPACE and its wide-field capabilities will enable us to map all the local massive SFRregs ([58] @ $<2 \mathrm{kpc}$ ) down to disk scales, and any SFReg of the Galaxy at resolutions $<0.1 \mathrm{pc}$.

In the mid-IR domain, surface brightness observations of massive SFRs will reveal the surface of clouds that are irradiated by local stars and by the interstellar radiation field (ISRF). Low-resolution (R 100-300) spectroscopic data in the 3-28 $\mu \mathrm{m}$ range will cover the emission of polycyclic aromatic hydrocarbons (PAHs) and very small grains (VSGs) which are mostly excited by far-UV photons. Past IR observatories have shown, on bright and nearby star-forming clouds, that the ratios of the aromatic spectral bands reflect the properties of the impinging radiation field. Along with the detection of the mid-IR $\mathrm{H}_{2}$ excitation lines, stringent constraints can be put on the impact of nearby young and massive stars on their parent molecular cloud. SPACE's wide-field and high-sensitivity performances will be crucial to generalize such analysis for many SFRs, since only the statistical comparison with star-formation indicators (e.g., the number and distribution of YSOs) will reveal the circumstances where SF feedback is positive or negative. Mid-IR $\mathrm{H}_{2}$ excitation lines will also allow us to study shocked regions and outflows that extend on scales beyond JWST mapping capabilities. These are important retroaction activities of star formation which are crucial to understand since they regulate the star formation efficiency of galaxies.

Mid-IR point sources are a goldmine for massive SFReg studies. Colour-magnitude diagnostic tools, coupled with total extinction determined from Herschel far-IR imaging from the Hi-GAL survey, can pinpoint the YSO age distributions in clumps via detailed comparison with model isochrones. Even simple colour-colour diagrams can be used for broad evolutionary classification of YSOs, determining the SF history in each stellar cluster. The power of SPACE in this respect is its ability to do this for tens and tens of degrees of the Galactic Plane, enabling critical comparative studies of young cluster formation as a function of their location in spiral arms, inter-arm regions, proximity to star-formation triggering agents like HII bubbles and relatively evolved $\mathrm{OB}$ associations.

Analysing the number and colour distributions of point sources will also enable us to compute high-resolution extinction maps of massive SFRs. Used in combination with current (UKIDSS, VVV) and coming (Roman Space Telescope) nearIR deep surveys, the longer wavelengths of SPACE will make it possible to detect background stars through the high-column density parts of the molecular clouds. 
Absorption of the background light in the $8 \mu \mathrm{m}$ to $20 \mu \mathrm{m}$ range also allows the deeply extinct parts to be studied with a resolution only limited by SPACE capabilities.

Finally, point-sources will enable us to characterize the dense and populous stellar clusters that form in massive SFRs. The study of their mass spectrum as a function of the environmental conditions is key to understand which processes determine the mass and multiplicity of stellar systems, and the Galactic star-formation history. However, such studies are usually hampered by the incompleteness of the stellar census. The wide-field and high-sensitivity performances of SPACE in the mid-IR domain will guarantee the completeness of the stellar census and of the mass distribution while including the youngest and most deeply embedded YSOs that remain invisible at near-IR wavelengths (Fig. 12).

This will be possible down to the low-mass end. Low mass stars are the faintest but also most numerous objects in the Galaxy. Their large number makes them the most profuse planetary systems, and recent observational evidence tend to show that they preferentially harbour low-mass, Earth-like planets, rather than giant planets, including in their habitable zone. SPACE will be perfectly suited to detect these stars to complete their census down to the substellar regime and to the planetary mass objects as well. The initial mass function (IMF) is an important constraint of theoretical and simulation work, so that a fine characterization of the IMF is one way to address the long-standing debate on whether the fragmentation of interstellar clouds into prestellar cores is dominated by gravitational fragmentation or by turbulent fragmentation. Recent studies demonstrated that, due to the core external pressure resulting from turbulence, low-mass stars and brown dwarfs can be formed more efficiently in the case of turbulent fragmentation than with gravitational fragmentation. The high sensitivity combined with the mid-IR range is well adapted to construct a sample with good statistics of these faint and red objects. The large field of view will also allow SPACE to probe a whole range of environments for many clouds. It will enable us to see statistically if clouds with active dynamics, (e.g. due to shock compression, or a high level of turbulence) are more prone to host lowmass object formation. Furthermore, the detection of the faint IR excesses around

Fig. 12 The Wide-Field Infrared Survey Explorer, or WISE, has uncovered a striking population of young stellar objects in a complex of dense, dark clouds in the southern constellation of Circinus. This mosaic from WISE covers an area on the sky so large that it could contain a grid of 11 by 7 full moons. The cloud itself is some 2,280 lightyears away and spans more than 180 light-years across. SPACE will observe such fields at subarcsec resolutions. Image credit: NASA/JPL-Caltech / UCLA

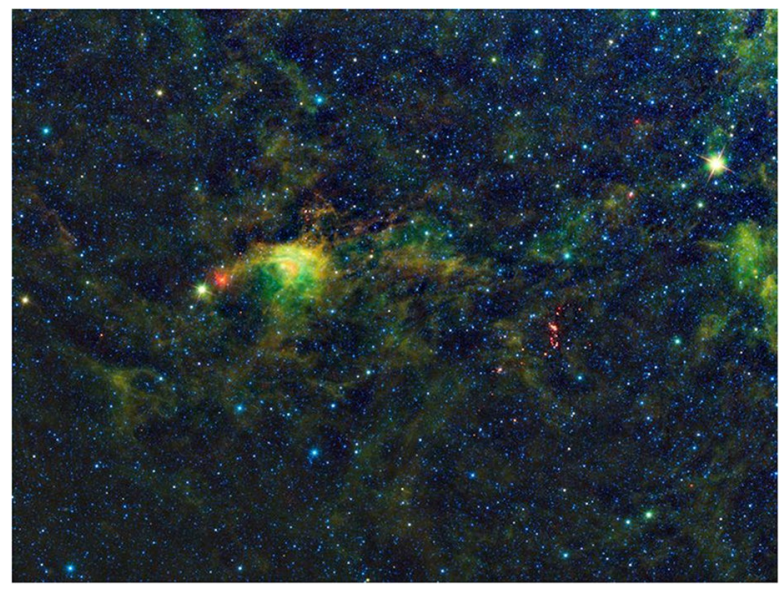


cool stars and brown dwarfs is a great challenge for upcoming instrumentation. Debris discs orders of magnitude fainter should be detectable around low-mass stars and brown dwarfs, up to a few hundred parsecs away. These observations will open new frontiers and will dramatically increase our knowledge of how these enigmatic objects are formed and what kind of planets they can harbour.

Grains have been shown to grow. Far-IR emission, compared to extinction, can better be explained if bigger grains are considered and the $9.8 \mu \mathrm{m}$ silicate or the $3 \mu \mathrm{m}$ water ice line widths seem to widen on some lines of sight, which is again attributable to grain growth. The most direct proof of this growth has been the discovery of coreshine, the scattering of MIR light in the 3-5 $\mathrm{m}$ range from cloud cores, and beyond that, the scattering up to $8 \mu \mathrm{m}$ that partly compensates the diffuse light extinction seen in that wavelength range towards cloud cores. Scattering is thought to be a strong tool to constrain grain properties, and it has become crucial to understand the optical behavior of dust from $1 \mu \mathrm{m}$ to $2000 \mu \mathrm{m}$. However, such models are still in their infancy and require deep and extensive data to be tested. Scattering is also seen as a tool to constrain the 3-dimensional (3D) structure of individual clouds when modelled from $1 \mu \mathrm{m}$ to $10 \mu \mathrm{m}$, each wavelength revealing a different layer of the clouds.

Grains get covered with ices as soon as the clouds start to be shielded from UV $\left(A_{V} \geq 1-3\right.$ mag). Water ice is the first to appear but among the abundant ices, $C O$ and $\mathrm{CO}_{2}$ are two important ones (Fig. 13). Today, we do not know how they evolve inside the clouds: whether they totally evaporate around the newborn star and if they do, up to which distance. They modify the grain properties and therefore have an impact on the measurement of the cloud mass in parallel with the grain growth, which they also facilitate by increasing the sticking efficiency and resistance to shocks in turbulent collisions.

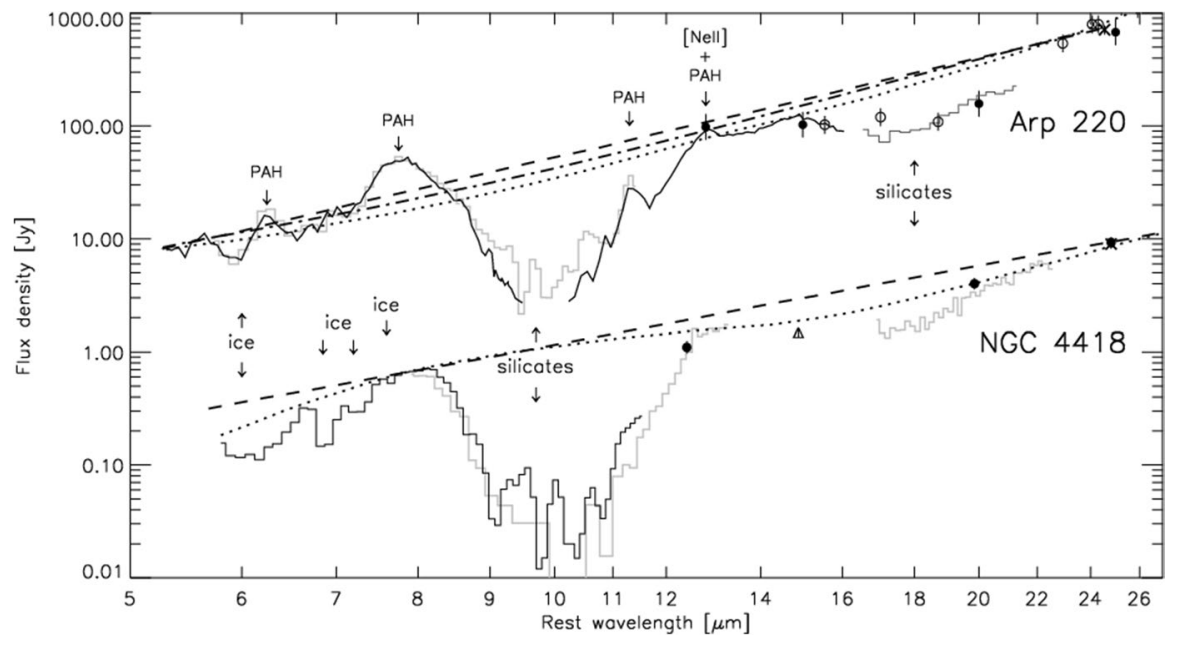

Fig. 13 From Spoon et al. [77]. Comparison of the mid-IR spectra of Arp 220 (multiplied by 90) and NGC 4418. The positions of absorption and emission bands are indicated, as are several choices for the local continuum for each object. Credit: H. Spoon, Cornell Univ 
It is now clear that progress in the cloud studies today need to constrain the grain properties in parallel with the cloud structure and mass. To achieve such progress both absorption and scattering in the near and mid-IR need to be measured with the highest possible sensitivity on large surfaces. Combining such observations with far-IR emission from Herschel observations, we will assess both the structure of the clouds and the grain composition and size distribution in 3D. This will open up 3D radiative transfer studies of molecular emission, 3D chemistry and, dynamical modelling. Like the Gaia project which, by measuring stellar astrometry feeds many branches of astrophysics in dire need of stellar distances, SPACE mapping of the interstellar clouds will provide the basic data we strongly need to get the basic knowledge on the clouds from which all the physics of interstellar clouds, including their chemistry, will be completely revisited.

In the coming years, before the launch of SPACE, such studies will be carried out with the material we can collect presently but neither near-IR data from groundbased telescopes nor Spitzer archive data are sensitive enough to allow for the kind of studies we want to perform in the end. We will certainly make strong progress on the grain properties and start to produce $3 \mathrm{D}$ cloud models but with a limited spatial resolution by lack of sensitivity. Gaia observations can also be used to trace the $3 \mathrm{D}$ structure of the outer parts of the clouds but since it is working in the visible, it will not go beyond a few magnitudes of extinction. Ground-based telescopes can hardly observe the brightest objects in the mid-IR wavelengths and only in a few narrow windows. Spitzer is now retired, and ground-based telescopes will always be limited by the sky glow. No improvement is expected before the launch of SPACE except the Roman Space telescope which will provide the NIR coverage and the latter is therefore not included in this project. The JWST is of course very sensitive but its field of view is far too small to map thousands of square degrees.

\section{Which science mission to address the science questions?}

The requirements derived from the science are summarized in Table 1 for a near-IR + mid-IR main instrument and in Table 2 for the (sub-)mm optional spectroscopic instrument.

With these requirements and with respect to the ESA Voyage 2050 programme, we have two options: one with a single standalone near-IR + mid-IR instrument in an M-sized mission. However, the science described in Sects. 1 and 2 would greatly benefit from a second option: a sub-mm instrument that would require an L-sized mission. This longer wavelength range allows us to see inside the dustier

Table 2 Summary of the requirements for the optional (sub-)millimetric spectroscopic instrument

\begin{tabular}{ll}
\hline Size of the primary mirror & $4-6 \mathrm{~m}$ \\
Wavelength range & $500-1000 \mu \mathrm{m}$ \\
Pixel scale spectroscopy & Diff. limited \\
Spectral resolution (Medium resolution) & 300 \\
Limiting line flux (1h, @ $800 \mu \mathrm{m}, \mathrm{SNR}=5)$ & A few $10^{-20} \mathrm{~W} / \mathrm{m}^{2}$ \\
\hline
\end{tabular}


regions and therefore contains a wealth of information on star formation. An all-sky survey to $3-5 \times 10^{-20} \mathrm{~W} / \mathrm{m}^{2}$ at $1 \sigma$ in the range $300-1000 \mu \mathrm{m}$ would open a unique discovery space for a statistical study and to discover exotic objects. A similar instrument could perform a deep survey of about 1000 hours over our 200 $\operatorname{deg}^{2}$ with a sensitivity of about $1.3 \times 10^{-20} \mathrm{~W} / \mathrm{m}^{2}$ at $800 \mu \mathrm{m}$.

\subsection{What if we assume an $M$ mission?}

SPACE could also be a standalone M mission. The near-IR + mid-IR instrumental design would still be constrained by the wide-field requirement which is mandatory for our science case. We could also consider that SPACE could be co-funded by another space agency to reduce the total cost and finally meet the cost cap requirements.

One very important option that is also mentioned in another White Paper (WP) is that an instrument similar to SPACE (specifically MISC) could be an ESA contribution to a NASA project like the Origins Space Telescope (Origins, [57]). Even though OST/MISC in the baseline design does not fully match our requirements, one of the OST up-scopes includes most of what we need to carry out the science described in this WP.

\subsection{What can we do with an L science mission?}

SPACE's scientific objectives would be better addressed if we assume an L mission with one imaging+spectroscopy instrument to cover the sky in the sub-mm and $\mathrm{mm}$ range. Such an instrument would allow candidate very high redshift galaxies to be identified over the entire sky, to be followed-up with the near-IR + mid-IR instrument or to be observed in parallel in near-IR + mid-IR while the telescope scans the sky.

Another option would be to build the mission in two phases. This would probably be more adapted to our science objectives. In the option described in Sect. 3.2, where we would share an $\mathrm{L}$ mission with a project observing the sky in the (sub-)mm like, e.g., PRISM (Primordial Radiation Imaging and Spectroscopy Mission), an all-sky survey will be carried out first until $\mathrm{T}=\mathrm{T}_{0}$. Then at $\mathrm{T}=\mathrm{T}_{1}$, the mission will focus on the ecliptic poles to perform a deeper survey. The Galactic Disc might also be covered to shallower magnitudes. The alternative would be to observe everything in a parallel mode. SPACE would be one of the instruments.

\subsection{Technology challenges}

Several technological challenges are identified to build a facility for SPACE's science. We provide details in the following of the section. 


\subsubsection{Wide-field NIR+MIR detectors}

In the NIR, up to about $5.3 \mu \mathrm{m}$, we have now detectors that are quite efficient and space-qualified. For instance, JWST uses two different types of detectors: 4Mpixels H2RG detectors for the 0.6-5 $\mu \mathrm{m}$ NIR (Teledyne Imaging Sensors) and 1Mpixel Si:As detectors for the 5-28 $\mu \mathrm{m}$ MIR (Raytheon Vision Systems). Both are built in California, therefore we need to explore potential providers in Europe.

\subsubsection{Lightweight large mirror}

For years the limit size for a lightweight space mirror, in the optical/IR range, was considered to be around $1.5 \mathrm{~m}-2.0 \mathrm{~m}$ in diameter, mainly due to the difficulty to maintain the right shape of the mirror with the required precision at these wavelengths. A $2.0 \mathrm{~m}+\varnothing$ rigid enough mirror has a significant mass that is a real drawback for a space mission while an ultra-lightweight mirror is too floppy to maintain the optical quality of the telescope after launch and during operations. During the last years, developments have been pursued world-wide to break this limit, mainly in two directions:

- Large SiC mirror with excellent optical quality that allow large lightweight rigid mirrors,

- In-flight correction of thermo-elastic and gravity-varying induced deformations of large lightweight mirrors using active loop with wave-front sensors and deformable mirrors.

European industry such as ADS/Mersen-Boostec has real expertise with the manufacturing of the $3.5 \mathrm{~m}$ mirror of the Herschel telescope [73], but excellent results have been published by Zhang et al. ([99], Fig. 14) demonstrating the manufacturing of a $4 \mathrm{~m} \mathrm{SiC} \mathrm{lightweight} \mathrm{mirror} \mathrm{with} \mathrm{a} \mathrm{surface} \mathrm{shape} \mathrm{better} \mathrm{than} \lambda / 30$ in optical wavelength $(\lambda=632.8 \mathrm{~nm})$. For future large missions, the technology for manufacturing large primary mirrors is ready and will be soon mature.

\subsubsection{Deformable mirror for active optics}

Only in Europe, many developments on deformable mirrors for space active-optics have been pursued during the past 10 years (Fig. 15. In Europe, ESA has recently funded a number of programmes to further develop Deformable Mirror (DM) technologies specifically for space applications. Münster University developed a smallformat Unimorph post-focal DM. As part of two independent active correction chain projects (i.e. WFSensing, DMs, Algorithms), two large monolithic deformable mirrors were also developed. The first, AOCC, was developed by TNO and the second, STOIC, by Fraunhofer IOF in collaboration with NUI Galway. Other European and national agencies have funded DM developments over the years; either to push forward new concepts or increase the Technology Readiness Level (TRL) of already developed and used technologies. In France, Thales-Alenia-Space (TAS) validated a new active DM, based on a concept defined with the Laboratoire d'Astrophysique 

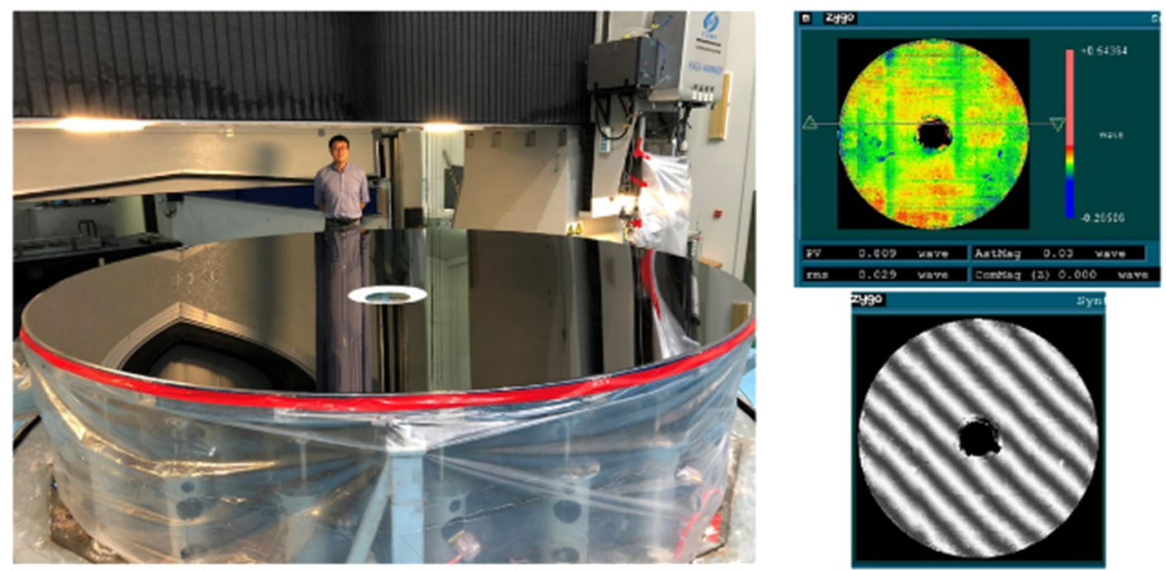

Fig. 14 Light-weight mirror by Ge Zhang et al. [99] that shows that the technology is making fast progress and suggests that our present assumptions are realistic objectives for the Voyage 2050 programme. Credit: G. Zhang / Changchun Institute of Optics, Fine Mechanics and Physics (CIOMP)

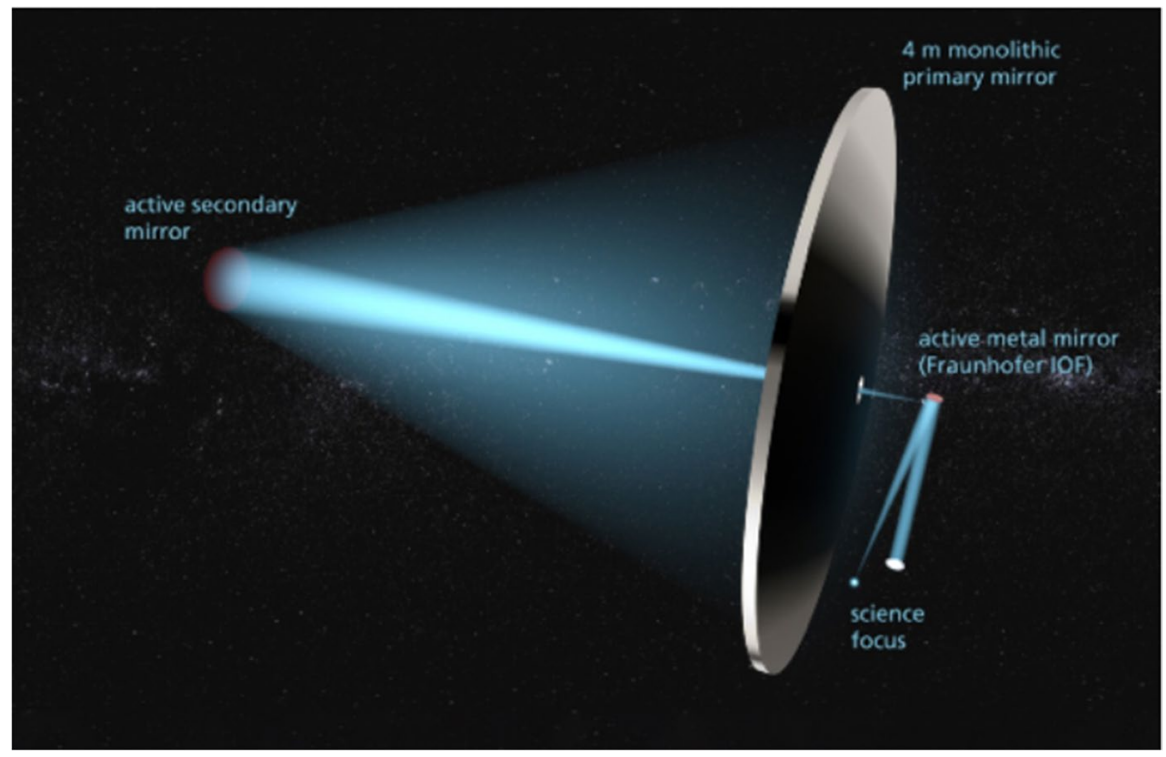

Fig. 15 Left: STOIC concept with $4 \mathrm{~m}$ monolithic mirror and active deformable mirrors $\odot$ Fraunhofer IOF. See: https://exoplanets.nasa.gov/exep/technology/TDEM-awards/). Right: MADRAS DM @ ThalesAlenia-Space \& LAM

de Marseile (LAM). This MADRAS DM, developed at TAS under CNES contract, sustains a launch environment without a locking device. The device has been fully integrated, and performance successfully verified. It is space qualified before assembly on the full-scale active-optic instrument demonstrator. 
Finally, a large number of traditional DM manufacturers are also present in Europe (e.g. Cilas, ADS-Microgate, Alpao, Imagine Optic). In the USA, NASA funded a number of DM technology developments for space applications, mainly through the Technology Development for Exoplanet Missions (TDEM, in particular companies such as, Boston Micromachines Inc (BMM) and Xinetic).

\subsubsection{Wide-field integral-field spectrograph}

Integral field spectroscopy has proved to be a very powerful tool in recent years for studies of the distant Universe using ground-based facilities. The combination of spatial and spectral information allows key diagnostics of galaxy formation and assembly to be probed, such as the kinematics of disk formation and the growth of galaxy bulges and black holes. Narrow-field integral field capabilities are planned for JWST, but the wide-field IR integral field capability proposed for SPACE is unique. It will open up a completely new discovery space which is inaccessible from the ground. In addition to the targeted spectroscopic surveys outlined in previous chapters, integral field spectroscopy has a unique potential for serendipitous discoveries by data-mining the resultant 3D data cubes for new emission-line sources invisible at other wavelengths. The technology for producing image slicers for integral field spectroscopy using diamond-machining techniques is now mature, and scalable from smaller field prototypes developed for NIRSpec and MIRI on JWST [48]. For instance, a method for cost efficient and high performant manufacturing of spherical image slicers was developed and patented in collaboration with the Winlight company. The technology was applied to VLT-MUSE instrument and is proposed for E-ELT and space instruments (Fig. 16).

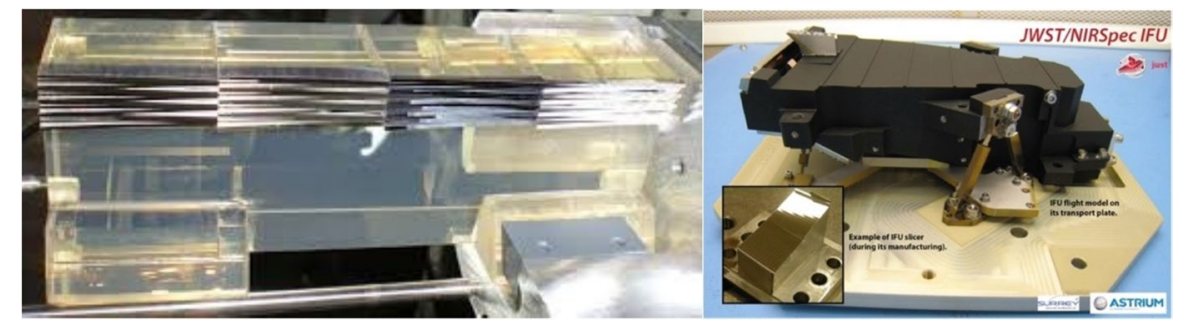

Fig 16 Left: The Image Dissector Array (IDA) manufacturing constitutes a WINLIGHT Optics/CNRS patent. Innovative methods, developed conjointly by LAM (Laboratoire d'Astrophysique de Marseille, France) and WinLight Optics (Marseille, France), allow high performances (accurate roughness, sharp edges, surface form, etc.) to be reached with standard glass manufactured components while saving costs and time by an order of magnitude compared with classical techniques. This IDA is composed of 48 slices. Right: Main picture: The NIRSpec IFU flight model. The IFU is roughly the size of a shoe box and weighs less than $1 \mathrm{~kg}$. Insert: A diamond-machined image slicer, a key element of the IFU, pictured during manufacture. Credit: R. Sharples / University of Durham, United Kingdom 


\subsubsection{Micro-mirror arrays}

The scientific return from future astrophysical space missions could be optimized using MOEMS (Micro-Opto-Electro-Mechanical systems) devices like large micromirror arrays (MMA). Multi-object spectrographs (MOS) are powerful tools for space and ground-based telescopes for the study of the formation and evolution of galaxies. This technique requires a programmable slit mask for astronomical object selection; 2D MMAs are suited for this task. MOEMS has been used to build JWST NIRSpec. In Europe, several options exist such as the one from Laboratoire d'Astrophysique de Marseille (LAM, France) and the Centre Suisse d'Electronique et de Microtechnologies (CSEM, Switzerland, who are engaged in a European development of MMAs, called MIRA, which is exhibiting remarkable performances in terms of surface quality as well as ability to work at cryogenic temperatures. MMA with $100 \times 200 \mu \mathrm{m}^{2}$ single-crystal silicon micromirrors were successfully designed, fabricated and tested down to $162 \mathrm{~K}$ (Fig. 17). They are designed to work at $30 \mathrm{~K}$ and there are no blocking points that would prevent them to work at $5 \mathrm{~K}$. In order to fill large focal planes (mosaicking of several chips), we are currently developing large micromirror arrays to be integrated with their electronics.

\section{Conclusion and roadmap}

We present in this paper a scientific project to study the early Universe, from the first stars to the end of the reionization. To meet the science objectives, we define a Space Project for Astrophysical and Cosmological Exploration (SPACE) as part of the ESA long term planning Voyage 2050 programme. SPACE will chart the

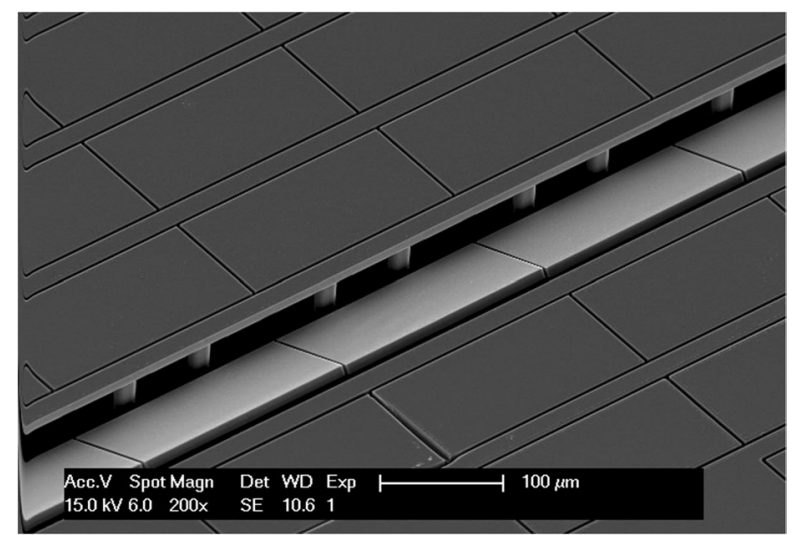

Fig. 17 Micro-mirror array with high fill factor in the long direction providing long slits [97]. Each mirror measures $200 \times 100 \mu \mathrm{m}^{2}$. Our project will take advantage of the already available building blocks to design, realize, and package customized micro-mirror arrays perfectly suited for our instruments. 2D arrays are built on wafer with Through Wafer Vias in order to allow routing of the device on wafer backside, foreseeing integration with dedicated ASICs. Like for CCDs, mosaicking will permit wide fields of view. Credit: F. Zamkotsian / LAM \& CSEM 
formation of the heavy elements, measure the evolution of the galaxy luminosity function, trace the build-up of stellar mass in galaxies over cosmic time, and find the first supermassive black holes (SMBHs) to form.

SPACE will provide us with a much larger sample of objects in the early Universe than any current or planned mission of very high redshift galaxies at $z>10$. These objects will be bright enough for a detailed follow-up spectroscopy.

To our knowledge, no single planned project can directly address the scientific objectives detailed in this paper for several reasons:

- To carry out the rise of metals science case, we must observe in the range $3-28 \mu \mathrm{m}$ to follow the bright rest-frame optical lines and/or the PAH3.3 $\mu \mathrm{m}$ feature. No other tracers can measure the metallicity of galaxies at $\mathrm{z} \gtrsim 5$ efficiently and reliably.

- For all the science cases described in this White Paper, no planned instrumental project is optimal. We need a field of view much wider that the ones provided by e.g., JWST, SPICA/SMI and even the present OST/MISC. On the other hand, AKARI and WISE all-sky surveys are not deep enough, and their angular resolutions are too low.

- Ground-based telescopes in the optical+NIR like the ELT, in the (sub-)mm like ALMA, NOEMA, and LMT are not competitive because of the wavelength range (ELT), the field of view (ALMA, NOEMA) and because spectroscopy in not integrated/developed (e.g. NIKA2, LMT). SPACE will be complementary to the SKA that will provide us with observations of anisotropies in the brightness temperature of the $21 \mathrm{~cm}$ line of neutral hydrogen from the period before reionization and would shed light on the dawn of the first stars and galaxies [70].

Author's contributions • Extragalactic Astrophysics and Cosmology (Andrew Bunker, Denis Burgarella, Rychard Bouwens, Jose Afonso, David Alexander, Hakim Atek, Karina Caputi, Laure Ciesla, Chris Conselice, Asantha Cooray, Giovanni Cresci, Jesus Gallego Maestro, Roberto Maiolino, Katarzyna Malek, Filippo Mannucci, Pascal Oesch, Chris Pearson, José Miguel Rodríguez Espinosa, Daniel Schaerer, David Rosario, David Sobral)

- Milky Way Studies: from the Global Structure to Dust Characterization (Laurent Pagani, Marc Audard, Julien Montillaud, Céline Reylé, Nadège Lagarde, Sylvie Cabrit, Isabelle Ristorcelli, Itsuki Sakon)

- Lightweight Large Mirror (Marc Ferrari)

- Deformable Mirror for Active Optics (Marc Ferrari)

- Wide-field Integral-Field Spectrograph (Ray Sharples)

- Micro-Mirror Arrays (Frédéric Zamkotsian)

Funding Available through the institutes where the co-authors institutes.

Data availability Not applicable

Code availability Not applicable

Declarations

Conflicts of interest Not applicable 
Open Access This article is licensed under a Creative Commons Attribution 4.0 International License, which permits use, sharing, adaptation, distribution and reproduction in any medium or format, as long as you give appropriate credit to the original author(s) and the source, provide a link to the Creative Commons licence, and indicate if changes were made. The images or other third party material in this article are included in the article's Creative Commons licence, unless indicated otherwise in a credit line to the material. If material is not included in the article's Creative Commons licence and your intended use is not permitted by statutory regulation or exceeds the permitted use, you will need to obtain permission directly from the copyright holder. To view a copy of this licence, visit http://creativecommons.org/licen ses/by/4.0/.

\section{References}

1. André, P. 2017CRGeo.349..187A

2. Amorin, R. et al. 2017NatAs...1E..52A

3. Bacon, R. et al. 2015A\&A...575A..75B

4. Baldwin, J.A, Philips, M.M., Terlevich, R. (1981PASP...93....5B)

5. Banados E. et al. (2018Natur.553..473B)

6. Barret et al. (2020AN....341..224B)

7. Behroozi, P.S., Wechsler, R.H. \& Conroy, C. (2013ApJ...770...57B)

8. Behroozi, Silk (2015ApJ...799...32B)

9. Bogdanoska, J., Burgarella, D. (2020MNRAS.496.5341B)

10. Boselli, A., Lequeux, J., Gavazzi, G. (2004A\&A...428..409B)

11. Bouwens, R.J., Illingworth, G.D., Gonzalez, V. et. al. (2010ApJ...725.1587B)

12. Bowler, R.A.A. et al. (2014MNRAS.440.2810B)

13. Bromm, V., Larson, R.B. (2004ARA\&A..42...79B)

14. Bunker, A.J. et al. (2004MNRAS.355..374B)

15. Bunker, A.J. et al. (2010MNRAS.409..855B)

16. Burgarella, D. et al. (2007MNRAS.380..986B)

17. Burgarella, D. et al. (2020A\&A...637A..32B)

18. Burgarella et al. (2016SPIE.9904E..2NB)

19. Calvi, R; Rodríguez Espinosa, J.M., Mas-Hesse, J.M., et al (2019MNRAS.489.3294C)

20. Caputi, K. et al. (2015ApJ...810...73C)

21. Castellano, M., Dayal, P., Pentericci, L., et al. (2016ApJ...818L...3C)

22. Ciesla, L. et al. (2014A\&A...565A.128C)

23. Content et al. 2013 (2013SPIE.8860E..0EC)

24. Curti, M. et al. (2017MNRAS.465.1384C)

25. Davé R. et al. (2011MNRAS.416.1354D)

26. De Barros, S. et al. (2019MNRAS.489.2355D)

27. Dijkstra, M. (2014PASA...31...40D)

28. Dopita, M.A. et al. (2016Ap\&SS.361...61D)

29. Duncan, K. et al. (2014MNRAS.444.2960D)

30. Dunlop, J.S. et al. (2012MNRAS.420..901D)

31. Durkalec, A. et al. (2015A\&A...583A.128D)

32. Feltre, A., Charlot, S., Gutkin, J. (2016MNRAS.456.3354F)

33. Fernández-Ontiveros, J.A. et al. (2016ApJS..226...19F)

34. Finkelstein, S.L. et al. (2015ApJ...814...95F)

35. Gaia Collaboration, Prusti, T. et al. (2016A\&A...595A...1G)

36. Galliano, E. et al. (2003A\&A...412..615G)

37. Gardner J.P. (2009ASPC..418..365G)

38. Gordon, J.D. et al. (2008ApJ...682..336G)

39. Grainge K. et al. (2017ARep...61..288G)

40. Harikane, Y. et al. (2016ApJ...821..123H)

41. Hartwig, T. et al. (2016MNRAS.462.2184H)

42. Iliev, I.T. et al. (2006MNRAS.369.1625I)

43. Ivezic Z. et al. (2019ApJ...873..111I) 
44. Kewley, L.J. et al. (2015ApJ...812L..20K)

45. Khostovan, A.A. et al. (2016MNRAS.463.2363K)

46. Lacey, C.G., et al. (2016MNRAS.462.3854L)

47. Lang, D. (2014AJ....147..108L)

48. Laurent, F. et al. (2004SPIE.5494..196L)

49. Laureijs R. J. et al. (2010SPIE.7731E..1HL)

50. Madden, S.C., et al. (2006A\&A...446..877M)

51. Madau, P., Dickinson, M. (2014ARA\&A..52..415M)

52. Maiolino R.,et al. (2008A\&A...488..463M)

53. Maiolino, R., Mannucci, F. (2019A\&ARv..27....3M)

54. Mannucci, F. et al. (2010MNRAS.408.2115M )

55. Mashian, N., Oesch, P.A., Loeb, M. (2016MNRAS.455.2101M)

56. Mason, C.A., Trenti, M., Treu, T. et al. (2015ApJ...813...21M)

57. Meixner, M., Cooray, A., Leisawitz, D. et al. (2019arXiv191206213M)

58. Ménard, B., et al. (2010MNRAS.405.1025M)

59. Mortlock, D.J., et al. (2011Natur.474..616M)

60. Nakajima, K., et al. (2016ApJ...831L...9N)

61. Nanni, A., et al. (2020arXiv200615146N)

62. Nomoto, K., et al. (2013ARA\&A..51..457N)

63. Oesch, P., et al. (2016ApJ...819..129O)

64. Oteo, I., et al. (2015MNRAS.452.2018O)

65. Ouchi, M., Shimasaku, K., Furusawa, H. et al. (2010ApJ...723..869O)

66. Pagel, B. (1979NewSc..82..902P)

67. Rodríguez Espinosa, J.M., Mas Hesse, J.M., et al. (2020MNRAS.495L..17R)

68. Robertson, B.E. et al. (2010Natur.468...49R)

69. Robertson, B.E., Ellis, R. S., Furlanetto, S. R, Dunlop, J.S. (2015ApJ...802L..19R)

70. Santos MG, Silva MB, Pritchard JR, Cen R, Cooray A (2011) Probing the first galaxies with the Square Kilometer Array. AAP 527:A93. https://doi.org/10.1051/0004-6361/201015695

71. Schaerer, D. (2003A\&A...397..527S)

72. Schaye, J. et al. (2015MNRAS.446..521S)

73. Sein, E. et al., (2003SPIE.4850..606S)

74. Shapiro, P.R., Giroux, M.L., Babul, A. (1994ApJ...427...25S)

75. Smit, R. et al. (2014ApJ...784...58S)

76. Sobral D. et al. (2015ApJ...808..139S)

77. Spoon, H.W.W. et al. (2004A\&A...414..873S)

78. Stacy, A., Bromm, V., Lee, A.T. (2016MNRAS.462.1307S)

79. Stark, D.P. et al. (2014MNRAS.445.3200S)

80. Stark, D.P. (2016ARA\&A..54..761S)

81. Steidel, C. C. et al. (1996AJ....112..352S)

82. Steinhardt, C.L. et al. (2016ApJ...824...21S)

83. Stutz, A.M. \& Gould A. (2016A\&A...590A...2S)

84. Taylor, P., Kobayashi, C. (2016MNRAS.463.2465T)

85. Tinsley, B. (1980A\&A....89..246T)

86. Tornatore, L., Ferrara, A., Schneider, R. (2007MNRAS.382.1050T)

87. Torrey, P. et al. (2018MNRAS.477L..16T)

88. Tremonti, C.A. et al. (2004ApJ...613..898T)

89. Venemans, B.P. et al. (2013ApJ...779...24V)

90. Visbal, E., Haiman, Z., Bryan, G.L. (2016MNRAS.460L..59V)

91. Vogelsberger, M. et al. (2014MNRAS.444.1518V)

92. Whalen, D.J. et al. (2013ApJ...777..110W)

93. Wilkins, S.M. et al. (2020MNRAS.493.6079W)

94. Wootten, A., Thompson, A.R. (2009IEEEP..97.1463W)

95. Yang, X. et al. (2006MNRAS.373.1159Y)

96. Zahid, H.J et al. (2011ApJ...730..137Z)

97. Zamkotsian, F. et al., "Large 1D and 2D micro-mirror arrays for Universe and Earth Observation", Proceedings of the SPIE conference on MOEMS 2019, Proc. SPIE 10931, (2019SPIE10931E..03Z)

98. Zehavi, I. et al. (2011 ApJ...736...59Z) 
99. Zhang, G. et al. Proc. SPIE 10837, 9th International Symposium on Advanced Optical Manufacturing and Testing Technologies: Large Mirrors and Telescopes, 108370I (2019SPIE10837E..0IZ)

Publisher's note Springer Nature remains neutral with regard to jurisdictional claims in published maps and institutional affiliations.

\section{Authors and Affiliations}

Denis Burgarella ${ }^{1}$ D Andrew Bunker ${ }^{2} \cdot$ Rychard Bouwens $^{3} \cdot$ Laurent Pagani $^{4}$. Jose Afonso ${ }^{5} \cdot$ Hakim Atek ${ }^{6}$. Marc Audard ${ }^{7}$. Sylvie Cabrit ${ }^{4} \cdot$ Karina Caputi $^{8}$. Laure Ciesla $^{1} \cdot$ Christopher Conselice $^{9} \cdot$ Asantha Cooray $^{10} \cdot$ Giovanni Cresci $^{11}$. Mirko Curti ${ }^{12}$. José Miguel Rodríguez Espinosa ${ }^{13}$ - Marc Ferrari ${ }^{1}$. Chiaki Kobayashi $^{14}$. Nadège Lagarde ${ }^{15}$. Jesus Gallego Maestro ${ }^{16}$. Roberto Maiolino ${ }^{12} \cdot$ Katarzyna Malek $^{17}$. Filippo Mannucci ${ }^{11}$. Julien Montillaud ${ }^{15}$. Pascal Oesch ${ }^{7}$. Chris Pearson ${ }^{18}$. Agnieszka Pollo ${ }^{17}$. Céline Reylé $^{15}$. David Rosario ${ }^{19}$. Itsuki Sakon ${ }^{20}$. Daniel Schaerer ${ }^{7}$. Ray Sharples $^{21}$ - David Sobral ${ }^{22}$. Frédéric Zamkotsian ${ }^{1}$

Denis Burgarella

denis.burgarella@lam.fr

Andrew Bunker

andy.bunker@physics.ox.ac.uk

1 Aix Marseille Univ, CNRS, CNES, LAM, Marseille, France

2 Department of Physics, University of Oxford, Keble Road, Oxford OX13RH, UK

3 Leiden Observatory, Leiden University, PO Box 9513, 2300 RA Leiden, The Netherlands

4 LERMA \& UMR8112 du CNRS, Observatoire de Paris, PSL University, Sorbonne Universités, CNRS, F-75014 Paris, France

5 Instituto de Astrofísica e Ciências do Espaço, Faculdade de Ciências, Universidade de Lisboa, OAL, Tapada da Ajuda, PT1349-018 Lisboa, Portugal

6 Sorbonne Université, CNRS UMR 7095, Institut d'Astrophysique de Paris, 98 bis bvd Arago, 75014 Paris, France

7 Observatoire de Geneve, Université de Geneve, 51 Ch. des Maillettes, 1290 Versoix, Switzerland

8 Kapteyn Astronomical Institute, University of Groningen, 9700 AV Groningen, The Netherlands

9 Centre for Astronomy and Particle Theory, University of Nottingham, University Park, Nottingham NG7 2RD, UK

10 University of California, Irvine, Irvine, CA, USA

11 INAF - Osservatorio Astrofisico di Arcetri, largo E. Fermi 5, 50127 Firenze, Italy

12 Kavli Institute for Cosmology - University of Cambridge, Madingley Road, Cambridge CB3 OHA, UK

13 Instituto de Astrofisica de Canarias (IAC), E-38205 La Laguna, Spain

14 School of Physics, Astronomy and Mathematics, Centre for Astrophysics Research, University of Hertfordshire, College Lane, Hatfield AL10 9AB, UK 
15 Institut UTINAM, CNRS UMR6213, Univ. Bourgogne Franche-niversidaComté, OSU THETA Franche-Comté-Bourgogne, Observatoire de Besançon, BP 1615, 25010 Besançon Cedex, France

16 Departamento de Física de la Tierra y Astrofísica, Facultad CC Físicas, Instituto de Física de Partículas y del Cosmos, IPARCOS, Universidad Complutense de Madrid, 28040 Madrid, Spain

17 National Centre for Nuclear Research, ul. Hoza 69, 00-681 Warszawa, Poland

18 RAL Space, STFC Rutherford Appleton Laboratory, Didcot, Oxfordshire OX11 0QX, UK

19 Centre for Extragalactic Astronomy, Department of Physics, Durham University, South Road, Durham DH1 3LE, UK

20 Department of Astronomy, Graduate School of Science, The University of Tokyo, Tokyo 113-0033, Japan

21 Center for Advanced Instrumentation, Department of Physics, Durham University, South Road, Durham DH1 3LE, UK

22 Department of Physics, Lancaster University, Lancaster LA1 4YB, UK 